\title{
The Effect of Side Chains on Competing Pathways for $\beta$ - Scission Reactions of Peptide-Backbone Alkoxyl Radicals
}

\author{
Geoffrey P. F. Wood, ${ }^{\dagger, \downarrow}$ Christopher J. Easton, ${ }^{\ddagger, 8}$ Arvi Rauk, ${ }^{\perp}$ Michael J.
}

Davies, ${ }^{\ddagger, t}$ and Leo Radom ${ }^{\dagger, t, *}$

School of Chemistry, University of Sydney, Sydney, NSW 2006, Australia, Research School of Chemistry, Australian National University, Canberra, ACT 0200, Australia, Department of Chemistry, University of Calgary, Calgary, AB, T2N IN4

Canada, Heart Research Institute, Camperdown, Sydney, NSW 2050, Australia, and Centre of Excellence in Free Radical Chemistry and Biotechnology

\section{SUPPORTING INFORMATION}

(Tables S1-S6, Total 22 pages)

\footnotetext{
*Email: radom@chem.usyd.edu.au

${ }^{\dagger}$ University of Sydney.

* Centre of Excellence in Free Radical Chemistry and Biotechnology.

$\S$ Australian National University.

${ }^{\perp}$ University of Calgary.

${ }^{\text {\# }}$ Heart Research Institute.
} 


\title{
TABLE S1: GAUSSIAN Archive Entries for B3-LYP/6-31G(d) Optimized Equilibrium Structures of Species Involved in Reactions A1-A6
}

\author{
$\mathrm{CH}_{2} \mathrm{OCHO}$ \\ $1 \backslash 1 \backslash$ GINC-SC9 \FOpt $\backslash$ UB3LYP $\backslash 6-31 G(d) \backslash C 2 H 3 O 2(2) \backslash G P W 501 \backslash 17-J u l-2002 \backslash 0 \backslash \backslash \#$ UB $3 L Y P / 6-31$ \\ $\mathrm{G}(\mathrm{D}) \mathrm{FOPT}=(\mathrm{CALCFC}, \mathrm{TIGHT}) \mathrm{GEOM}=\mathrm{ALLCHECK}$ MAXDISK $=131072000 \mathrm{SCF}=(\mathrm{MAXC}$ \\ $\mathrm{YC}=100)$ GUESS=READ $\backslash \mathrm{H}-\mathrm{C}(=\mathrm{O})-\mathrm{C}\left(\mathrm{HO}^{*}\right)-\mathrm{H} \mathrm{c} 1$ frequency $\backslash \backslash 0,2 \backslash \mathrm{O}, 1.741073 \quad 756,-0.06331616$ \\ $85,-0.2177858557 \backslash \mathrm{C}, 0.677621205,0.3662802515,0.1444895394 \backslash \mathrm{H}, 0.5262794287,1.400442266$ \\ $3,0.5153797066 \backslash \mathrm{C},-0.6172566902,-0.48932156 \quad 49,0.13970897 \backslash \mathrm{H},-0.4705289606,-1.396149726$ \\ $6,-0.4714123984 \backslash \mathrm{O},-1.705817269,0.2558826315,-0.1514491232 \backslash \mathrm{H},-0.6999894522,-0.80657636$ \\ 34,1.20472146 7\\Version=DEC-AXP-OSF/1-G98RevA.11.3\HF $=-228.3666921 \backslash \mathrm{S} 2=0.753664 \backslash \mathrm{S}$ \\ $2-1=0 . \backslash \mathrm{S} 2 \mathrm{~A}=0.75001 \backslash \mathrm{RMSD}=9.204 \mathrm{e}-09 \backslash \mathrm{RMSF}=1.825 \mathrm{e}-06 \backslash \mathrm{Dipole}=-0.0852206,-0.1269906,0.5$ \\ $374159 \backslash \mathrm{PG}=\mathrm{C} 01[\mathrm{X}(\mathrm{C} 2 \mathrm{H} 3 \mathrm{O} 2)] \backslash \backslash @$ \\ $\mathrm{CH}_{2} \mathrm{O}-\mathrm{CHO}$
}

$1 \backslash 1 \backslash$ GINC-SC113\FTS \UB3LYP $\backslash 6-31 G(d) \backslash C 2 H 3 O 2(2) \backslash G P W 501 \backslash 13-J a n-2003 \backslash 0 \backslash \backslash \#$ B 3LYP/6-31 G(D) OPT $=($ TS,NOEIGENTEST,TIGHT,MAXCYC $=100)$ MAXDISK=134217728 FREQ $\backslash$ ts $\backslash \backslash 0$, $2 \backslash \mathrm{O},-0.7953870311,0 .,-1.7949678351 \backslash \mathrm{C},-0.7961039582,0 .,-0.6172557375 \backslash \mathrm{C}, 0.9693180461,0 ., 0$. $6059905911 \backslash \mathrm{O}, 0.544785788,0 ., 1.7669427458 \backslash \mathrm{H}, 1.3170965876,-0.9299527557,0.1091104149 \backslash \mathrm{H}$ $, 1.3170965876,0.9299527557,0.1091104149 \backslash \mathrm{H},-1.6686677578,0 ., 0.0735707632 \backslash \backslash$ Version=DECAXP-OSF $/ 1-G$ 98RevA.11.3 $\backslash$ State $=2-A^{\prime} \backslash \mathrm{HF}=-228.3493227 \backslash \mathrm{S} 2=0.763066 \backslash \mathrm{S} 2-1=0 . \backslash \mathrm{S} 2 \mathrm{~A}=0.75004$ $6 \backslash \mathrm{RMSD}=5.398 \mathrm{e}-09 \backslash \mathrm{RMSF}=2.205 \mathrm{e}-06 \backslash \mathrm{Dipole}=-0.3343358,0 .,-0.5921043 \backslash \mathrm{PG}=\mathrm{CS}[\mathrm{SG}(\mathrm{C} 2 \mathrm{H} 1 \mathrm{O} 2)$ ,X(H2)]\\@

$\mathrm{CH}_{2} \mathrm{O}$

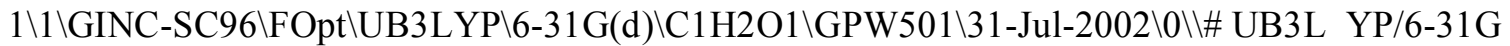
* FOPT $=$ TIGHT GEOM $=$ CHECKPOINT SCF=TIGHT MAXDISK $=655360000 \backslash \mathrm{H} 2 \mathrm{C} *=\mathrm{O}$ geo metry optimisation $\backslash \backslash 0,1 \backslash \mathrm{O}, 0 ., 0 .-0.6775712819 \backslash \mathrm{C}, 0 ., 0 ., 0.52890318 \quad 87 \backslash \mathrm{H}, 0.9377829383,0 ., 1.1235$ $755616 \backslash \mathrm{H},-0.9377829383,0 ., 1.1235755616 \backslash \backslash$ Ver sion=DEC-AXP-OSF/1-G98RevA.11.3 $\backslash$ State $=1$ $-\mathrm{A} 1 \backslash \mathrm{HF}=-114.5004726 \backslash \mathrm{S} 2=0 . \backslash \mathrm{S} 2-1=0 . \backslash \mathrm{S} 2 \mathrm{~A}=0 . \backslash \mathrm{RMSD}=5.908 \mathrm{e}-09 \backslash \mathrm{RMSF}=1.911 \mathrm{e}-06 \backslash \mathrm{Dipole}=0$. $0 ., 0.8601887 \backslash \mathrm{PG}=\mathrm{C} 02 \mathrm{~V}[\mathrm{C} 2(\mathrm{C} 1 \mathrm{O} 1), \mathrm{SGV}(\mathrm{H} 2)] \backslash \backslash @$

\section{$\mathrm{CHO}$}

$1 \backslash 1 \backslash$ GINC-SC19\FOpt $\backslash U B 3 L Y P \backslash 6-31 G(d) \backslash C 1 H 1 O 1(2) \backslash G P W 501 \backslash 17-J u l-2002 \backslash 0 \backslash \backslash \#$ B 3LYP/6-31( D) $\mathrm{FOPT}=\mathrm{TIGHT}$ GEOM=CHECKPOINT $\mathrm{SCF}=\mathrm{TIGHT}$ MAXDISK $=131072000 \backslash \mathrm{H} \mathrm{C}^{*}=\mathrm{O}$ geo metry optimisation $\backslash \backslash 0,2 \backslash \mathrm{C},-0.1350226676,0 .,-0.576862179 \backslash \mathrm{O}, 0.01 \quad 12535837,0 ., 0.5967859643 \backslash \mathrm{H}$, $0.7201073361,0 .,-1.3131146403 \backslash \backslash$ Version=DEC- AXP-OSF $/ 1-G 98 R e v A .11 .3 \backslash$ State $=2-A^{\prime} \backslash H F=-1$ $13.8501711 \backslash \mathrm{S} 2=0.752491 \backslash \mathrm{S} 2-1=0 . \backslash \mathrm{S} 2 \mathrm{~A}=0.750004 \backslash \mathrm{RMSD}=1.630 \mathrm{e}-09 \backslash \mathrm{RMSF}=4.042 \mathrm{e}-06 \backslash \mathrm{Dipole}$ $=0.2852608,0 .,-0.541573 \backslash \mathrm{PG}=\mathrm{CS}[\mathrm{SG}(\mathrm{C} 1 \mathrm{H} 1 \mathrm{O} 1)] \backslash \backslash @$

\section{$\mathrm{CH}_{3} \mathrm{CHOCHO}$}

$1 \backslash 1 \backslash$ GINC-SC122\FOpt $\backslash U B 3 L Y P \backslash 6-31 G(d) \backslash C 3 H 5 O 2(2) \backslash G P W 501 \backslash 15-J u l-2003 \backslash 0 \backslash \backslash \#$ B3LYP/6-31 $\mathrm{G}^{*} \mathrm{OPT}=\mathrm{TIGHT}$ FREQ MAXDISK $=52428800 \mathrm{SCF}=\mathrm{TIGHT} \backslash \backslash 5 \backslash \backslash 0,2 \backslash \mathrm{O},-1.09 \quad 99300612,0.13792$ $99899,-1.4859712605 \backslash \mathrm{C},-0.8778937203,-0.4994923879,-0.4 \quad 930062552 \backslash \mathrm{C}, 0.4165215156,-0.30$ $57884713,0.3808820761 \backslash \mathrm{O}, 0.0146348023,-0 . \quad 3152810975,1.6691121749 \backslash \mathrm{H},-1.5591577542,-1.2$ 
$752841427,-0.093882752 \backslash \mathrm{H}, 0 \quad .9323444019,-1.2730409529,0.1666074768 \backslash \mathrm{C}, 1.27139796,0.878$ $6400214,-0.06 \quad 17241678 \backslash \mathrm{H}, 1.5490946662,0.7953801249,-1.1167814701 \backslash \mathrm{H}, 2.1805066321,0.91$ $87247981,0.5453176828 \backslash \mathrm{H}, 0.7194195932,1.8128740604,0.0767018294 \backslash \backslash$ Versio $n=D E C-A X P-$ $\mathrm{OSF} / 1-\mathrm{G} 03 \mathrm{RevB} .03 \backslash \mathrm{State}=2-\mathrm{A} \backslash \mathrm{HF}=-267.6880656 \backslash \mathrm{S} 2=0.754465 \backslash \mathrm{S} 2-1=0 . \backslash \mathrm{S} 2 \mathrm{~A}=0.750013 \backslash \mathrm{RMS}$ $\mathrm{D}=2.615 \mathrm{e}-09 \backslash \mathrm{RMSF}=1.822 \mathrm{e}-06 \backslash$ Dipole $=0.3700212,-0.4322 \quad 51,-0.1833405 \backslash \mathrm{PG}=\mathrm{C} 01[\mathrm{X}(\mathrm{C} 3 \mathrm{H} 5 \mathrm{O}$ 2)]\\@

$\mathrm{CH}_{3} \mathrm{CHO}-\mathrm{CHO}$ (Transition Structure (TS))

$1 \backslash 1 \backslash$ GINC-LC2\FTS \UB3LYP $\backslash 6-31 G(d) \backslash C 3 H 5 O 2(2) \backslash G P W 501 \backslash 19-A p r-2005 \backslash 0 \backslash \backslash \#$ B3L YP/6-31G (D) OPT $=($ NOEIGENTEST,TS,CALCFC) MAXDISK $=671088640$ FREQ $=$ NORAMAN $\|$ comm net $\backslash 0,2 \backslash \mathrm{O},-1.5393499665,-1.2150140006,0.2130763089 \backslash \mathrm{C},-0.5924873933,-1.0820167313,-0.47$ $90848212 \backslash \mathrm{C}, 0.6670624243,0.5699971156,-0.3613690703 \backslash \mathrm{O}, 1.6271776315,-0.0861492056,0.09$ $23560558 \backslash \mathrm{H},-0.001829904,-1.870858 \quad 0697,-0.9900041905 \backslash \mathrm{H}, 0.5984680702,0.7560713562,-1.4$ $561973033 \backslash \mathrm{C},-0.1333999812,1.5371126076,0.4903715897 \backslash \mathrm{H},-1.1335427509,1.7076163851,0.0$ $797370556 \backslash \mathrm{H}, 0.3967664764,2.4985563511,0.5075021808 \backslash \mathrm{H},-0.2095335108,1.1673616756,1$. $5159971497 \backslash \backslash$ Version $=I A 32 L-G 03 R e v C .02 \backslash$ State $=2-A \backslash H F=-267.6743506 \backslash \mathrm{S} 2=0.763024 \backslash \mathrm{S} 2-1=0$. $\backslash \mathrm{S} 2 \mathrm{~A}=0.750048 \backslash \mathrm{RMSD}=3.509 \mathrm{e}-09 \backslash \mathrm{RMSF}=1.727 \mathrm{e}-05 \backslash \mathrm{Dipole}=-0.4962922,0.1641457,-0.621081$ $2 \backslash \mathrm{PG}=\mathrm{C} 01[\mathrm{X}(\mathrm{C} 3 \mathrm{H} 5 \mathrm{O} 2)] \backslash \backslash @$

\section{$\mathrm{CH}_{3} \mathrm{CHO}$}

$1 \backslash 1 \backslash$ GINC-SC33\FOpt $\backslash R B 3 L Y P \backslash 6-31 G(d) \backslash C 2 H 4 O 1 \backslash G P W 501 \backslash 06-D e c-2002 \backslash 0 \backslash \backslash \#$ B3LY P/6-31G* FREQ MAXDISK $=134217728$ OPT $=$ TIGHT $\backslash \backslash$ comf $1 \backslash \backslash 0,1 \backslash \mathrm{O},-0.4971152259,0 .,-1.167043149 \backslash \mathrm{C}$, $-0.4611618032,0 ., 0.0429842474 \backslash \mathrm{C}, 0.8037340058,0 ., 0.8645184521 \backslash \mathrm{H}, 1.6818006162,0 ., 0.21475$ $39651 \backslash \mathrm{H}, 0.8218646594,0.8808207315,1.5199355377 \backslash \mathrm{H}, 0.8218646594,-0.8808207315,1.519935$ $5377 \backslash \mathrm{H},-1.4040413439,0 ., 0.6367039544 \backslash \backslash$ Version=DEC-AXP-OSF/1-G98RevA.11.3 \State=1-A' $\backslash \mathrm{HF}=-153.8301195 \backslash \mathrm{RMSD}=7.561 \mathrm{e}-09 \backslash \mathrm{RMSF}=2.170 \mathrm{e}-06 \backslash \mathrm{Dipole}=0.2173139,0 ., 1.0160585 \backslash \mathrm{P} \mathrm{G}=$ CS [SG(C2H2O1),X(H2)]\\@,

\section{$\mathrm{CH}_{3} \mathrm{CHOCONH}_{2}$}

$1 \backslash 1 \backslash$ GINC-SC8\FOpt $\backslash U B 3 L Y P \backslash 6-31 G(d) \backslash C 3 H 6 N 1 O 2(2) \backslash G P W 501 \backslash 10-J a n-2003 \backslash 0 \backslash \backslash \# \quad$ B3LYP/6-3 $1 \mathrm{G}(\mathrm{D}) \mathrm{OPT}=(\mathrm{TIGHT}, \mathrm{MAXCYC}=100)$ FREQ MAXDISK=134217728 $\backslash \mathrm{comf} 1 \backslash \backslash 0,2 \backslash \mathrm{H}, 1.676840$ $6121,-1.6478578856,0.3241555931 \backslash \mathrm{C}, 1.8028356636,-0.663585306,-0.1343997658 \backslash \mathrm{C}, 0.707540$ $7922,0.2969962187,0.3241996938 \backslash \mathrm{O}, 0.73661720 \quad 1,1.5307587041,-0.2121338035 \backslash \mathrm{H}, 1.7739997$ $708,-0.7783946419,-1.222377048 \quad 1 \backslash \mathrm{H}, 2.7798988942,-0.2573147583,0.144721612 \backslash \mathrm{C},-0.752867$ $2572,-0.3187218901,0.0629610121 \backslash \mathrm{O},-0.9222651327,-1.5209278208,0.0437923678 \backslash \mathrm{H}, 0.68369$ $10384,0.3676580436,1.434739599 \backslash \mathrm{N},-1.7010674865,0.6281134655,-0.068203383 \quad 7 \backslash \mathrm{H},-2.6585$ $130588,0.3576858051,-0.2428959349 \backslash \mathrm{H},-1.4083165883,1.5946479765,-0.1307542907 \backslash \backslash$ Version $=$ DEC-AXP-OSF $/ 1-G 98 R e v A .11 .3 \backslash \mathrm{HF}=-323.0773086 \backslash \mathrm{S} 2=0.754714 \backslash \mathrm{S} 2-1=0 . \mathrm{S} 2 \mathrm{~A}=0.750013 \backslash \mathrm{R}$ $\mathrm{MSD}=9.752 \mathrm{e}-09 \backslash \mathrm{RMSF}=1.862 \mathrm{e}-06 \backslash$ Dipole $=-0.5329643,0.4746963,0.2079815 \backslash \mathrm{PG}=\mathrm{C} 01[\mathrm{X}(\mathrm{C} 3 \mathrm{H}$ 6N1O2)]\@

\section{$\mathrm{CH}_{3} \mathrm{CHO}--\mathrm{CONH}_{2}$ (TS)}

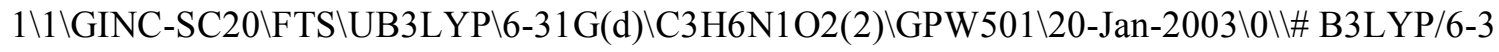
$1 \mathrm{G}(\mathrm{D})$ OPT $=(\mathrm{TS}$, NOEIGENTEST,TIGHT,MAXCYC $=100)$ MAXDISK=134217728 FREQ $\backslash \backslash$ ts $\backslash \backslash 0$ $, 2 \backslash \mathrm{H},-1.7648651378,0.9190735129,-1.11818916 \backslash \mathrm{C},-1.7664697239,0.9325969416,-0.024447777$ $9 \backslash \mathrm{C},-0.3573232892,0.9509239851,0.5366426002 \backslash \mathrm{O},-0.0872793176,0.531181789,1.680935165 \backslash$ $\mathrm{H},-2.3174138235,0.0704522189,0.3618939744 \backslash \mathrm{H},-2.2822836475,1.8446259785,0.3061094294 \backslash$ 
$\mathrm{C}, 0.5885585971,-0.5681955542,-0.664774172 \backslash \mathrm{O}, 0.4111830257,-0.6702378444,-1.8463969725 \backslash$ $\mathrm{H}, 0.3209608778,1.6847245498,0.0497892197 \backslash \mathrm{N}, 1.3494855502,-1.2031200897,0.2153750309 \backslash$ $\mathrm{H}, 1.9484994941,-1.9767989249,-0.0506749449 \backslash \mathrm{H}, 1.2688802164,-0.8997404997,1.182616823 \backslash$ Version=DEC-AXP-OSF/1-G98RevA.11.3 \HF=-323 .0665405 $\backslash \mathrm{S} 2=0.760485 \backslash \mathrm{S} 2-1=0 . \mathrm{IS} 2 \mathrm{~A}=0.75$ $004 \backslash \mathrm{RMSD}=8.064 \mathrm{e}-09 \backslash \mathrm{RMSF}=2.021 \mathrm{e}-06 \backslash \mathrm{Dipole}=0.4943266,-0.4383047,-0.0200519 \backslash \mathrm{PG}=\mathrm{C} 01[$ X(C3H6N1O2)]\\@,

$\mathrm{CONH}_{2}$

$1 \backslash 1 \backslash$ GINC-SC9\FOpt $\backslash U B 3 L Y P \backslash 6-31 \mathrm{G}(\mathrm{d}) \backslash \mathrm{C} 1 \mathrm{H} 2 \mathrm{~N} 1 \mathrm{O} 1(2) \backslash \mathrm{GPW} 501 \backslash 06-J a n-2003 \backslash 0 \backslash \backslash \#$ B3LYP/6-31 $\mathrm{G}^{*} \mathrm{OPT}=(\mathrm{TIGHT}, \mathrm{MAXCYC}=100)$ MAXDISK $=134217728$ FREQ GEOM=CHECK GUESS $=\mathrm{R}$ $\mathrm{EAD} P O P=\mathrm{NO} \backslash \backslash$ comf $3 \backslash \backslash 0,2 \backslash \mathrm{N},-0.3546345427,0 .,-1.0280399367 \backslash \mathrm{C},-0.3146790567,0 ., 0.3165703$ $468 \backslash \mathrm{O}, 0.6392413441,0 ., 1.0489505903 \backslash \mathrm{H}, 0.4965149049,0 .,-1.5859390775 \backslash \mathrm{H},-1.2399295184,0 .,-$ $1.5088081691 \backslash \backslash$ Version=DEC-AXP-O SF/1-G98RevA.11.3 $\backslash$ State $=2-A^{\prime} \backslash H F=-169.2320624 \backslash \mathrm{S} 2=0$. $75272 \backslash \mathrm{S} 2-1=0 . \backslash \mathrm{S} 2 \mathrm{~A}=0.750005 \backslash \mathrm{RMSD}=5.596 \mathrm{e}-09 \backslash \mathrm{RMSF}=2.594 \mathrm{e}-06 \backslash \mathrm{Dipole}=-0.4272591,0 .,-1.3$ 606678\P G=CS [SG(C1H2N1O1)] \\

\section{$\mathrm{CHONHCOCH}_{3} \mathrm{CHO}$}

$1 \backslash 1 \backslash$ GINC-SC105\FOpt $\backslash U B 3 L Y P \backslash 6-31 G(d) \backslash C 4 H 6 N 1 O 3(2) \backslash G P W 501 \backslash 09-D e c-2002 \backslash 0 \backslash \backslash \#$ B3LYP/ 6-31G* OPT $=($ TIGHT,MAXCYC $=200)$ FREQ MAXDISK $=26214400 \backslash \backslash 6 \backslash 10,2 \backslash \mathrm{O},-1.9253112863$, $-0.1456671553,-0.8730403326 \backslash \mathrm{C},-1.9252926845,-0.145642148,0.3516398547 \backslash \mathrm{N},-0.7939477809$ $,-0.1456510596,1.1013718765 \backslash \mathrm{H},-0.8399467249,-0.0026705782,2.1033713206 \backslash \mathrm{H},-2.854005873$ $2,-0.1425674157,0.9513742391 \backslash \mathrm{C}, 0.5589381335,-0.1420069085,0.5145214233 \backslash \mathrm{O}, 1.456933799$, $0.3519845061,1.3282201821 \backslash \mathrm{O}, 1.2006885331,0.9406052964,-1.6596854144 \backslash \mathrm{C}, 0.535132729,1$. $0179073289,-0.67861447 \backslash \mathrm{H},-0.0895376003,1.8787934426,-0.3878564298 \backslash \mathrm{H}, 1.0057185177,-2.2$ $446578723,0.6205967727 \backslash \mathrm{C}, 0.9116678211,-1.4813488991,-0.157185112 \backslash \mathrm{H}, 1.8640910473,-1.37$ $15613237,-0.6784497924 \backslash \mathrm{H}, 0.1301507392,-1.7666162521,-0.8647649019 \backslash \backslash$ Version=DEC-AXPOSF/1-G98RevA.11.3\HF=-4 36.3849876 $\backslash \mathrm{S} 2=0.759946 \backslash \mathrm{S} 2-1=0 . \mathrm{S} 2 \mathrm{~A}=0.750031 \backslash \mathrm{RMSD}=5.727 \mathrm{e}-$ 09\RMSF=1.156e -06\Dipole=-0.6331824,-0.0701419,1.0949922\PG=C01 [X(C4H6N1O3)]\\@,

\section{$\mathrm{CHONHCOCH}_{3}-\mathrm{CHO}$ (TS)}

$1 \backslash 1 \backslash G I N C-S C 22 \backslash F T S \backslash U B 3 L Y P \backslash 6-31 G(d) \backslash C 4 H 6 N 1 O 3(2) \backslash G P W 501 \backslash 30-J a n-2003 \backslash 0 \backslash \backslash \# \quad B 3 L Y P / 6-$ $31 \mathrm{G}(\mathrm{D}) \mathrm{OPT}=(\mathrm{TS}$, NOEIGENTEST,TIGHT,MAXCYC=100) $\mathrm{MAXDISK}=134217728$ FREQ IO $\mathrm{P}(1 / 8=10) \backslash \backslash \mathrm{ts} \backslash \backslash 0,2 \backslash \mathrm{O},-0.9952062931,0.6876712177,-2.0496652995 \backslash \mathrm{C},-1.01687224,0.614219033$ $1,-0.875986293 \backslash \mathrm{C}, 0.6620195594,0.5930236341,0 . \quad 2550423959 \backslash \mathrm{H},-1.8652375409,0.7971684931$

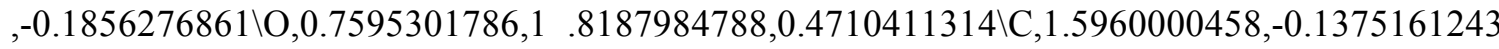
,-0.7014418306\O, -0.8363665909,-1.9006241288,0.2164953878\C,-0.5298976083,-1.36660439 $89,1.2728174243 \backslash \mathrm{N}, 0.1859260608,-0.2094002158,1.3756558781 \backslash \mathrm{H}, 0.185909496,0.28430075$ $07,2.2619776668 \backslash \mathrm{H},-0.8214861641,-1.7810748865,2.2557242961 \backslash \mathrm{H}, 2.5770992816,-0.2076778$ $818,-0.216702859 \backslash \mathrm{H}, 1.6967237555,0.4477236202,-\quad 1.6172304277 \backslash \mathrm{H}, 1.2343518474,-1.1401359$ 913,-0.9332920726 $\backslash$ Version=DEC-A XP-OSF/1-G98RevA.11.3\HF=-436.380776 $\backslash \mathrm{S} 2=0.760733$ $\backslash \mathrm{S} 2-1=0 . \backslash \mathrm{S} 2 \mathrm{~A}=0.750037 \backslash \mathrm{RMSD}=4.287 \mathrm{e}-09 \backslash \mathrm{RMSF}=4.030 \mathrm{e}-06 \backslash \mathrm{Dipole}=-0.1679941,-0.1662531$, $1.1033421 \backslash \mathrm{P} G=\mathrm{C} 01[\mathrm{X}(\mathrm{C} 4 \mathrm{H} 6 \mathrm{~N} 1 \mathrm{O} 3)] \backslash \backslash @$

\section{$\mathrm{CHONHCOCH}_{3}$}

$1 \backslash 1 \backslash$ GINC-SC88\FOpt $\backslash R B 3 L Y P \backslash 6-31 G(d) \backslash C 3 H 5 N 1 O 2 \backslash G P W 501 \backslash 10-D e c-2002 \backslash 0 \backslash \backslash \#$ B3 LYP/6-31 $\mathrm{G}^{*} \mathrm{OPT}=(\mathrm{TIGHT}, \mathrm{MAXCYC}=100)$ FREQ MAXDISK $=134217728 \backslash \backslash$ meconhcho $\backslash \backslash 0,1 \backslash \mathrm{H},-1.52803$ $75337,0 .,-1.1778600177 \backslash \mathrm{C},-1.5449637635,0 .,-0.0786646734 \backslash \mathrm{N},-0.2679183244,0.0 .4715457779$ 
$\backslash \mathrm{C}, 0.9160351555,0 .,-0.2770866354 \backslash \mathrm{O}, 0.9057412443,0 .,-1.4922230265 \backslash \mathrm{C}, 2.1797358965,0 ., 0.56$ $0833594 \backslash \mathrm{H}, 3.0427926442,0 .,-0.1055361726 \backslash \mathrm{H}, 2.2191369929,0.8856961005,1.2060468857 \backslash \mathrm{H}, 2$. $2191369929,-0.8856961005,1.2060468857 \backslash \mathrm{H},-0.2288851868,0 ., 1.4852090727 \backslash \mathrm{O},-2.549936165$ $5,0 ., 0.5990704252 \backslash \backslash$ Version=DEC-AXP-OSF $/ 1-G 98 R$ RevA.11.3 $\backslash$ State $=1-A^{\prime} \backslash H F=-322.5466453 \backslash \mathrm{R}$ $\mathrm{MSD}=3.619 \mathrm{e}-09 \backslash \mathrm{RMSF}=4.809 \mathrm{e}-06 \backslash \mathrm{Dipole}=1.1164562,0 ., 0.8727547 \backslash \mathrm{PG}=\mathrm{CS}[\mathrm{SG}(\mathrm{C} 3 \mathrm{H} 3 \mathrm{~N} 1 \mathrm{O} 2)$, $\mathrm{X}(\mathrm{H} 2)] \backslash \backslash @$

\section{$\mathrm{CHONHCOCH}_{3} \mathrm{CONH}_{2}$}

$1 \backslash 1 \backslash$ GINC-AC45\FOpt $\backslash U B 3 L Y P \backslash 6-31 G(d) \backslash C 4 H 7 N 2 O 3(2) \backslash G P W 501 \backslash 05-A p r-2006 \backslash 0 \backslash \backslash \#$ b3lyp/6-3 $1 \mathrm{G}(\mathrm{d})$ opt freq $\backslash \backslash$ comment $\backslash 0,2 \backslash \mathrm{C},-0.099839431,1.065884479,0.3152260563 \backslash \mathrm{C},-1.3585481277,0$. $1258802842,-0.2285195858 \backslash \mathrm{H}, 2.820563792,-0.5 \quad 650505805,-0.584258193 \backslash \mathrm{C}, 1.8333806992,-0$. $5472942488,-0.0854083045 \backslash \mathrm{N}, 1.1414229632,0.5906865842,-0.361821335 \backslash \mathrm{O}, 1.4524408617,-1$. $4852811811,0.6037380197 \backslash \mathrm{H}, 1.6028520646,1.258489376,-0.9667802625 \backslash \mathrm{O},-0.1301443157,0.8$ $780828133,1.6164094539 \backslash \mathrm{C},-0.3766663079,2.5055394343,-0.139952724 \backslash \mathrm{H},-0.4829392521,2$. $5583666075,-1.2270467547 \backslash \mathrm{H}, 0.4416886803,3.152594649,0.1928 \quad 473758 \backslash \mathrm{H},-1.3064314795,2.8$ $433622609,0.318168241 \backslash \mathrm{O},-2.1967479217,0.6062805537,-0.9600340435 \backslash \mathrm{N},-1.3026875278,-1$. $1266687638,0.2476148886 \backslash \mathrm{H},-0.4332965125,-1.4487400231,0.6822317654 \backslash \mathrm{H},-1.950888185,-1$ $.8012222448,-0.1395145978 \backslash \backslash$ Version=IA64L-G03RevD.01 $\backslash$ State $=2-A \backslash H F=-491.7708532 \backslash \mathrm{S} 2=0$ $.755798 \backslash \mathrm{S} 2-1=0 . \backslash \mathrm{S} 2 \mathrm{~A}=0.750016 \backslash \mathrm{RMSD}=5.363 \mathrm{e}-09 \backslash \mathrm{RMSF}=2.444 \mathrm{e}-05 \backslash \mathrm{Thermal}=0 . \backslash \mathrm{Dipo} \mathrm{l}=0.6$ 480015,0.3153101,-0.9926039\PG=C01 [X(C4H7N2O3)]\\@

$\mathrm{CHONHCOCH}_{3}-\mathrm{CONH}_{2}$ (TS)

$1 \backslash 1 \backslash$ GINC-SC66 $\backslash$ FTS $\backslash$ UB3LYP $\backslash 6-31 \mathrm{G}(\mathrm{d}) \backslash \mathrm{C} 4 \mathrm{H} 7 \mathrm{~N} 2 \mathrm{O} 3(2) \backslash G P W 501 \backslash 03-D e c-2003 \backslash 0 \backslash$ \# B3LYP/6-3 $1 \mathrm{G}(\mathrm{D}) \mathrm{OPT}=(\mathrm{TIGHT}, \mathrm{MAXCYC}=100, \mathrm{TS}, \mathrm{CALCFC}, \mathrm{NOEIGENTEST}) \mathrm{MAXDISK}=52428800 \mathrm{~S}$ $\mathrm{CF}=($ TIGHT,MAXCYC=100) FREQ $\|$ comment $\backslash \backslash 0,2 \backslash \mathrm{O},-1.5603769198,-0.8304157952,-1.40976$ $00018 \backslash \mathrm{C},-1.3749150993,-0.6088429854,-0.2419896462 \backslash \mathrm{C},-0.1642197577,0.7011699169,0.2302$ $719329 \backslash \mathrm{O},-0.1903166282,0.6900108529,1.5064053304 \backslash \mathrm{N},-1.8905625155,-1.1582407198,0.8518$ $956342 \backslash \mathrm{H},-1.575007309,-0.7906134292,1.7433589384 \backslash \mathrm{H},-2.5541551553,-1.920053568,0.78748$ $2419 \backslash \mathrm{H},-0.8779816006,1.7242903126,-1.5726013652 \backslash \mathrm{C},-0.7538679059,1.9190686788,-0.5038$ $795764 \backslash \mathrm{H},-1.7215275076,2.173489289,-0.0646498027 \backslash \mathrm{H},-0.068650193,2.7614223293,-0.36432$ $34961 \backslash \mathrm{N}, 1.0381453075,0.2162767691,-0.4138944295 \backslash \mathrm{O}, 3.0880012582,-0.789233252,-0.330225$ $0564 \backslash \mathrm{C}, 2.1236511419,-0.3144315061,0.242660025 \backslash \mathrm{H}, 1.0523353344,0.143179199,-1.42570354$ $27 \backslash \mathrm{H}, 2.0295549316,-0.242645548,1.3366898273 \backslash \backslash$ Version=DEC-AXP-OSF/1-G03RevB.03 \Stat $\mathrm{e}=2-\mathrm{A} \backslash \mathrm{HF}=-491.7705169 \backslash \mathrm{S} 2=0.758039 \backslash \mathrm{S} 2-1=0 . \backslash \mathrm{S} 2 \mathrm{~A}=0.750028 \backslash \mathrm{RMSD}=7.937 \mathrm{e}-09 \backslash \mathrm{RMSF}=1.56$ 7e-06\Dipole=-1.7917705,0.0044388,0.3664204\PG=C01 [X(C4H7N2O3)]\\@,

\section{$\mathrm{CH}_{3} \mathrm{CONHCOCH}_{3} \mathrm{CONHCH}_{3}$}

$1 \backslash 1 \backslash$ GINC-SC103\FOpt $\backslash U B 3 L Y P \backslash 6-31 G(d) \backslash C 6 H 11 N 2 O 3(2) \backslash G P W 501 \backslash 13-J a n-2004 \backslash 0 \backslash \backslash \#$ B3LYP /6-31G(D) OPT $=($ TIGHT,MAXCYC $=100)$ MAXDISK $=65536000 \mathrm{SCF}=($ TIGHT,M AXCYC $=1$ 00) FREQ $\backslash \backslash 5 \backslash \backslash 0,2 \backslash C,-0.1016567335,1.0763503203,0.3146757769 \backslash \mathrm{C},-1 \quad .3765380348,0.15330304$ $22,-0.2092319995 \backslash \mathrm{C}, 3.2485003206,-0.5842211654,-0 \quad .6535756153 \backslash \mathrm{C}, 1.8466008739,-0.5274315$ $725,-0.0662915955 \backslash \mathrm{N}, 1.1341888224,0.6003147818,-0.3684226217 \backslash \mathrm{H}, 3.4951334784,-1.624392$ $2948,-0.8757157101 \backslash \mathrm{H}, 3.3618682389,0.0192826337,-1.560017706 \backslash \mathrm{O}, 1.4171163548,-1.443799$ $0199,0.633701484 \backslash \mathrm{H}, 1.5857780495,1.2785753982,-0.9671417771 \backslash \mathrm{O},-0.1278327153,0.896366$ $092,1.6186038926 \backslash \mathrm{C},-0.3715033927,2.5186564389,-0.1402664351 \backslash \mathrm{H},-0.4782886345,2.573287$ $2478,-1.2272833198 \backslash \mathrm{H}, 0.4508195524,3.1610667152,0.1923579163 \backslash \mathrm{H},-1.2992219273,2.861230$ $5124,0.3181393052 \backslash \mathrm{O},-2.2345161377,0.6492141075,-0.9134861421 \backslash \mathrm{N},-1.3214279967,-1.1038$ $006148,0.2484623308 \backslash \mathrm{H},-0.441052027,-1.3872526439,0.6906723575 \backslash \mathrm{C},-2.2767974112,-2.110$ 
$9591432,-0.1880089225 \backslash \mathrm{H},-1.9868014881,-2.5647343337,-1.1442631153 \backslash \mathrm{H},-2.3332065365,-2$ $.8941508238,0.5719297333 \backslash \mathrm{H}, 3.9638874442,-0.220166754,0.0932404502 \backslash \mathrm{H},-3.2580156778,-1$. $6467817844,-0.3065572276 \backslash \backslash$ Version=DEC-AXP-OSF $/ 1 \quad-$ G03RevB.03 $\backslash$ State $=2-A \backslash H F=-570.406$ $4334 \backslash \mathrm{S} 2=0.755655 \backslash \mathrm{S} 2-1=0 . \mathrm{S} 2 \mathrm{~A}=0.750016 \backslash \mathrm{RMSD}=8.146 \mathrm{e}-09 \backslash \mathrm{RMSF}=1.569 \mathrm{e}-06 \backslash \mathrm{Dipole}=0.866$ $849,0.0803088,-0.9770008 \backslash \mathrm{PG}=\mathrm{C} 01[\mathrm{X}(\mathrm{C} 6 \mathrm{H} 11 \mathrm{~N} 2 \mathrm{O} 3)] \backslash \backslash @$

\section{$\mathrm{CH}_{3} \mathrm{CONHCOCH}_{3}-\mathrm{CONHCH}_{3}$ (TS)}

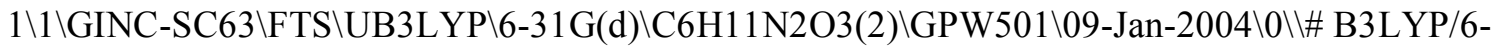
$31 \mathrm{G}(\mathrm{D}) \mathrm{OPT}=(\mathrm{CALCFC}, \mathrm{TS}, \mathrm{NOEIGENTEST}$,TIGHT,MAXCYC $=100)$ MAXDISK $=52428800$ $\mathrm{SCF}=(\mathrm{TIGHT}, \mathrm{MAXCYC}=100) \mathrm{FREQ} \backslash \backslash$ comment $\backslash \backslash 0,2 \backslash \mathrm{C}, 0.1043601538,1.2069923167,0.419857$ $1868 \backslash \mathrm{C},-1.4613919896,0.1617988368,-0.3023965025 \backslash \mathrm{C}, 3.1438250528,-0.8251341959,-0.75860$ $27685 \backslash \mathrm{C}, 1.8151922391,-0.6057924471,-0.0504431848 \backslash \mathrm{N}, 1.2058885933,0.5956178592,-0.32708$ $32178 \backslash \mathrm{H}, 3.3005396412,-1.8971793296,-0.8914923086 \backslash \mathrm{H}, 3.1980833105,-0.3239774594,-1.7310$ $179171 \backslash \mathrm{O}, 1.3493582068,-1.4467953601,0.711298997 \backslash \mathrm{H}, 1.6019307017,1.1542817891,-1.07164$ $88162 \backslash \mathrm{O},-0.0279763904,0.9469861043,1.6357153365 \backslash \mathrm{C},-0.2158267173,2.5941791003,-0.13387$ $69222 \backslash \mathrm{H},-0.3839729574,2.5821925407,-1.2142309614 \backslash \mathrm{H}, 0.6173211497,3.2683328686,0.10116$ $18763 \backslash \mathrm{H},-1.114248896,2.967290971,0.3590978093 \backslash \mathrm{O},-2.2067165024,0.6804075803,-1.0940594$ $572 \backslash \mathrm{N},-1.441121596,-1.0313034856,0.2717117266 \backslash \mathrm{H},-0.6115160181,-1.2412861084,0.8319591$ $363 \backslash \mathrm{C},-2.3799990453,-2.0956987479,-0.0756929246 \backslash \mathrm{H},-1.9172817211,-2.8302816708,-0.7449$ $105526 \backslash \mathrm{H},-2.7085910988,-2.6026768219,0.8361156617 \backslash \mathrm{H}, 3.9548370206,-0.4364462179,-0.131$ $2325398 \backslash \mathrm{H},-3.2447507858,-1.6533069491,-0.5729092646 \backslash \backslash$ Version=DEC-AXP-OSF/1-G03Rev B.03 $\backslash$ State $=2-\mathrm{A} \backslash \mathrm{HF}=-570.4022024 \backslash \mathrm{S} 2=0.761114 \backslash \mathrm{S} 2-1=0 . \backslash \mathrm{S} 2 \mathrm{~A}=0.75004 \backslash \mathrm{RMSD}=3.326 \mathrm{e}-09 \backslash \mathrm{RM}$ $\mathrm{SF}=2.960 \mathrm{e}-06 \backslash \mathrm{Dipole}=0.653$ 8046,-0.1636406,-1.2019348\} \backslash \mathrm { PG } = \mathrm { C } 0 1 \text { [X(C6H11N2O3)]\\@, }

\section{$\mathrm{CH}_{3} \mathrm{CONHCOCH}_{3}$}

$1 \backslash 1 \backslash$ GINC-SC160\FOpt $\backslash$ RB3LYP $\backslash 6-31 G(d) \backslash C 4 H 7 N 1 O 2 \backslash G P W 501 \backslash 09-D e c-2003 \backslash 0 \backslash \backslash \#$ B 3LYP/6$31 \mathrm{G}(\mathrm{D}) \mathrm{OPT}=(\mathrm{TIGHT}, \mathrm{MAXCYC}=100) \mathrm{MAXDISK}=65536000 \mathrm{SCF}=(\mathrm{TIGHT}, \mathrm{MAXCY} \mathrm{C}=100)$ FREQ $\backslash$ lcomment $\backslash 0,1 \backslash \mathrm{C},-2.4094943464,-0.8865345023,0 . \backslash \mathrm{C},-1.2634116411,0.1176422413,0 . \backslash$ $\mathrm{N}, 0.0000176498,-0.5018591491,0 . \backslash \mathrm{C}, 1.263411134,0.1 \quad 177102601,0 . \backslash \mathrm{H},-3.3511776629,-0.33684$ $73817,0.1 \mathrm{O},-1.4270614849,1.3157019164,0 . \mathrm{H}_{\mathrm{N}} 0.0000523837,-1.5140973641,0 . \backslash \mathrm{O}, 1.426991824$ 2,1.3157804186,0. \C,2.4095255832,-0.8864474666,0.।H,2.3694621096,-1.5312725102,-0.8863 $99951 \backslash \mathrm{H}, 2.3694621096,-1.5312725102,0.886399951 \backslash \mathrm{H}, 3.3512075931,-0.336730 \quad 1514,0 . \backslash \mathrm{H},-2$. $3693785874,-1.5314239571,-0.8863650764 \backslash \mathrm{H},-2.3693785874,-1 \quad .5314239571,0.8863650764 \backslash \backslash \mathrm{V}$

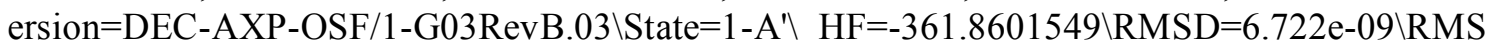

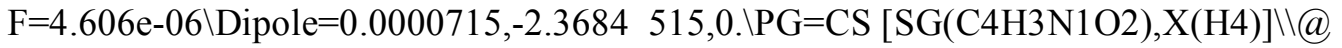

\section{$\mathrm{CONHCH}_{3}$}

$1 \backslash 1 \backslash$ GINC-SC77\FOpt $\backslash U B 3 L Y P \backslash 6-31 G(d) \backslash C 2 H 4 N 1 O 1(2) \backslash G P W 501 \backslash 28-N o v-2003 \backslash 0 \backslash \backslash \#$ B3LYP/6$31 \mathrm{G}(\mathrm{D}) \mathrm{OPT}=($ TIGHT,MAXCYC $=100)$ MAXDISK $=39321600 \mathrm{SCF}=($ TIGHT,MAX CYC $=100)$ FREQ $\backslash \backslash$ comment $\backslash 0,2 \backslash \mathrm{N}, 0.788287424,0.1136816294,0 . \backslash \mathrm{C},-0.24064613,0.9780867854,0 . \backslash \mathrm{O},-1.42$ $57424397,0.7544964968,0 . \mathrm{IC}, 0.6405199532,-1.3447821333,0 . \mathrm{H}, 1.7183540002,0.5056520003$, $0 . \mathrm{H},-0.4274518839,-1.5671229285,0 . \backslash \mathrm{H}, 1.0988912472,-1.785050182,-0.8925127656 \backslash \mathrm{H}, 1.0988$ $912472,-1.785050182,0.8925127656 \backslash \backslash$ Version=DEC-AXP-OSF $/ 1-G 03 R e v B .03 \backslash$ State $=2-A^{\prime} \backslash \mathrm{HF}=-$ $208.5432193 \backslash \mathrm{S} 2=0.752834 \backslash \mathrm{S} 2-1=0 . \backslash \mathrm{S} 2 \mathrm{~A}=0.750005 \backslash \mathrm{RMSD}=9.042 \mathrm{e}-09 \backslash \mathrm{RMSF}=3.148 \mathrm{e}-06 \backslash \mathrm{Dipol}$ $\mathrm{e}=1.3115479,-0.6930148,0 . \mathrm{PG}=\mathrm{CS}[\mathrm{SG}(\mathrm{C} 2 \mathrm{H} 2 \mathrm{~N} 1 \mathrm{O} 1), \mathrm{X}(\mathrm{H} 2)] \backslash \backslash$ 


\title{
TABLE S2: GAUSSIAN Archive Entries for B3-LYP/6-31G(d) Optimized Equilibrium Structures of Additional Species Involved in Reactions B1-B6
}

\author{
$\mathrm{CH}_{2} \mathrm{ONH}_{2}$

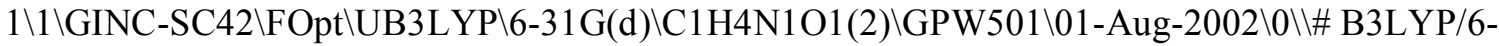 \\ $31 \mathrm{G}(\mathrm{D}) \mathrm{FOPT}=$ TIGHT $\mathrm{SCF}=$ TIGHT MAXDISK $=52428800$ GUESS $=($ READ) GEO $\mathrm{M}=\mathrm{CHECK}$ \\ POINT $\backslash \mathrm{O} *-\mathrm{C}(\mathrm{H} 2)-\mathrm{NH} 2$ optimisation AA $\backslash \backslash 0,2 \backslash \mathrm{H},-0.9135625135,0.8155560489,-1.176707901 \backslash \mathrm{N}$ \\ ,-0.3025958058,0.,-1.1642032855\C,0.4819443216,0.,0. 0522743562\H,1.1598706568,0.876193 \\ $4395,0.0456260823 \backslash \mathrm{H}, 1.1598706568,-0.8761934395,0.0456260823 \backslash \mathrm{O},-0.158263947,0 ., 1.26224$ \\ $25624 \backslash \mathrm{H},-0.9135625135,-0.8155560489,-1.176707901 \backslash \backslash$ Version=DEC-AXP-OSF/1-G98RevA.1 \\ $1.3 \backslash \mathrm{State}=2-\mathrm{A} " \backslash \mathrm{HF}=-170.4056145 \backslash \mathrm{S} 2=0.753041 \backslash \mathrm{S} 2-1=0 . \backslash \mathrm{S} 2 \mathrm{~A}=0.750007 \backslash \mathrm{RMSD}=5.354 \mathrm{e}-09 \backslash \mathrm{R}$ \\ $\mathrm{MSF}=5.300 \mathrm{e}-06 \backslash \mathrm{Dipole}=-0.0370889,0 .,-0.2715906 \backslash \mathrm{PG}=\mathrm{CS}[\mathrm{SG}(\mathrm{C} 1 \mathrm{~N} 1 \mathrm{O} 1), \mathrm{X}(\mathrm{H} 4)] \backslash \backslash @$ \\ $\mathrm{CH}_{2} \mathrm{O}-\mathrm{NH}_{2}(\mathrm{TS})$
}

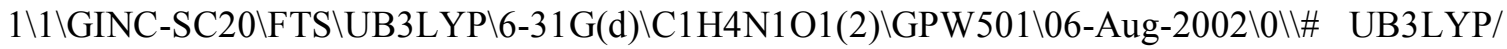
6-31G* OPT $=(\mathrm{TS}$, TIGHT,CALCFC) GEOM=CHECKPOINT MAXDISK=65536000 I NT(GRI $\mathrm{D}=\mathrm{ULTRAFINE}) \backslash \backslash \mathrm{H} 2-\mathrm{N}--\mathrm{C}(\mathrm{H} 2)-\mathrm{O} * \mathrm{TS}$ optimisation $\backslash \backslash 0,2 \backslash \mathrm{O},-0.7177253565,0 .,-0.9537147618 \backslash$ $\mathrm{C},-0.8009741534,0 ., 0.2729912703 \backslash \mathrm{N}, 1.3178202337,0 ., 0.6036295422 \backslash \mathrm{H}, 1.6033684589,0.80735$ $62836,0.0295785288 \backslash \mathrm{H}, 1.6033684589,-0.8073562836,0.0295785288 \backslash \mathrm{H},-0.9419153905,-0.9303$ $373747,0.85360331 \backslash \mathrm{H},-0.9419153905,0.9303373747,0.85360331 \backslash \backslash$ Version=DEC-AXP-OSF/1 G98RevA.11 .3\State $=2-A^{\prime} \backslash \mathrm{HF}=-170.3771455 \backslash \mathrm{S} 2=0.763086 \backslash \mathrm{S} 2-1=0 . \backslash \mathrm{S} 2 \mathrm{~A}=0.750062 \backslash \mathrm{RMSD}=9$.

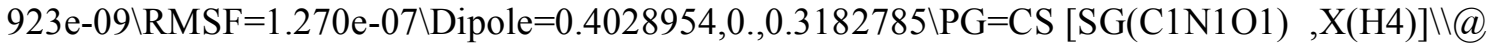

$\mathrm{NH}_{2}$

$1 \backslash 1 \backslash G I N C-S C 47 \backslash F O p t \backslash U B 3 L Y P \backslash 6-31 G(d) \backslash H 2 N 1(2) \backslash G P W 501 \backslash 19-J u l-2002 \backslash 0 \backslash \backslash \#$ B3L YP/6-31G* FOPT $=$ TIGHT MAXDISK $=65536000 \backslash \backslash \mathrm{N}^{*} \mathrm{H} 2$ optimisation $\backslash \backslash 0,2 \backslash \mathrm{N},-0.1184818129,0 .,-0.0826540$ $575 \backslash \mathrm{H},-0.0456206405,0 ., 0.9491237909 \backslash \mathrm{H}, 0.8749933305,0 .,-0.3705453884 \backslash \backslash$ Version=DEC-AXP -OSF/1-G98RevA.11.3 \State $=2-\mathrm{B} 1 \backslash \mathrm{HF}=-55.8726187 \backslash \mathrm{S} 2=0.752803 \backslash \mathrm{S} 2-1=0 . \backslash \mathrm{S} 2 \mathrm{~A}=0.750004 \backslash \mathrm{RM}$ $\mathrm{SD}=6.175 \mathrm{e}-09 \backslash \mathrm{RMSF}=1.120 \mathrm{e}-06 \backslash \mathrm{Dipole}=0.6493283,0 ., 0.4529777 \backslash \mathrm{PG}=\mathrm{C} 02 \mathrm{~V}[\mathrm{C} 2(\mathrm{~N} 1), \mathrm{SGV}(\mathrm{H} 2)$ ]1\@

\section{$\mathrm{CH}_{3} \mathrm{CHONH}_{2}$}

$1 \backslash 1 \backslash$ GINC-SC92\FOpt $\backslash U B 3 L Y P \backslash 6-31 G(d) \backslash C 2 H 6 N 1 O 1(2) \backslash G P W 501 \backslash 19-N o v-2002 \backslash 0 \backslash \backslash \#$ B3LYP/6

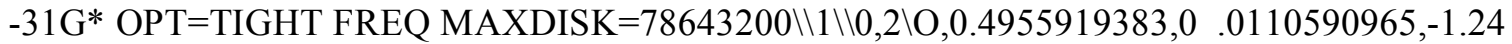
$83099084 \backslash \mathrm{C}, 0.3826152089,0.0380122647,0.1190859127 \backslash \mathrm{H}, 1 \quad .3568283635,-0.0184558957,0.63$ $31898305 \backslash \mathrm{N},-0.3033728018,-1.2472910084,0 \quad .3589187841 \backslash \mathrm{H},-1.1881950315,-1.2536673887,-0$. $1508075597 \backslash \mathrm{H}, 0.2630313231,-2.0150096414,-0.0020746139 \backslash \mathrm{C},-0.440027866,1.202372163,0.6$ $555018111 \backslash \mathrm{H}, 0.055856348,2.1483678395,0.4153259289 \backslash \mathrm{H},-0.5526387948,1.1280202168,1.7$ 42370131\H,-1.4315321595,1.2110025899,0.1885177195\\Version=DEC-AXP-O SF/1-G98Rev A. $11.3 \backslash \mathrm{HF}=-209.7275161 \backslash \mathrm{S} 2=0.754979 \backslash \mathrm{S} 2-1=0 . \mathrm{S} 2 \mathrm{~A}=0.750013 \backslash \mathrm{RMS} \mathrm{D}=7.492 \mathrm{e}-09 \backslash \mathrm{RMSF}=1.6$ 92e-06\Dipole=-0.2324551,-0.2665662,0.4142142\PG=C $01[\mathrm{X}(\mathrm{C} 2 \mathrm{H} 6 \mathrm{~N} 1 \mathrm{O} 1)] \backslash \backslash @$

$\mathrm{CH}_{3} \mathrm{CHO}-\mathrm{NH}_{2}$ (TS)

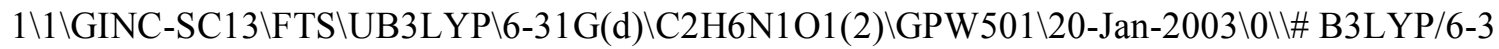
$1 \mathrm{G}(\mathrm{D})$ OPT $=(\mathrm{TS}$, NOEIGENTEST,MAXCYC $=100$,TIGHT) $\mathrm{MAXDISK}=134217728$ FREQ $\backslash \backslash$ ts $\backslash \backslash 0$ 
, $2 \backslash \mathrm{O}, 0.359437255,0.4558237454,-1.1883076515 \backslash \mathrm{C}, 0.3609937862,0.4519024626,0.0535465918 \backslash$ $\mathrm{H}, 1.3204873733,0.4546562346,0.6070578853 \backslash \mathrm{N}, 0.2297013038,-1.6084533846,0.1426297568 \backslash$ $\mathrm{H},-0.5673485877,-1.7772841793,-0.4884494858 \backslash \mathrm{H}, 1.0419482447,-1.8939580914,-0.422818068$ $9 \backslash \mathrm{C},-0.8410896179,0.8609025286,0.8771004611 \backslash \mathrm{H},-0.8007464947,1.9489890803,1.023249380$ $3 \backslash \mathrm{H},-1.7672479226,0.6228620762,0.3464416376 \backslash \mathrm{H},-0.8299247894,0.3804886612,1.858689248$ $8 \backslash \backslash$ Version=DEC-AXP-OSF/1-G98RevA.11.3\HF=-209.7029542 $\backslash \mathrm{S} 2=0.765879 \backslash \mathrm{S} 2-1=0 . \backslash \mathrm{S} 2 \mathrm{~A}=0$. $750075 \backslash \mathrm{RMSD}=4.170 \mathrm{e}-09 \backslash \mathrm{RMSF}=1.254 \mathrm{e}-06 \backslash \mathrm{Dipole}=-0.1153572,-0.2912855,0.3946105 \backslash \mathrm{PG}=\mathrm{C} 0$ $1[\mathrm{X}(\mathrm{C} 2 \mathrm{H} 6 \mathrm{~N} 1 \mathrm{O} 1)] \backslash \backslash$

\section{$\mathrm{CHONHCHOCH}_{3}$}

$1 \backslash 1 \backslash$ GINC-SC103\FOpt $\backslash U B 3 L Y P \backslash 6-31 G(d) \backslash C 3 H 6 N 1 O 2(2) \backslash G P W 501 \backslash 18-D e c-2002 \backslash 0 \backslash \backslash$ \# B3LYP/

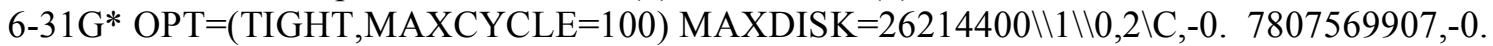
$0802033725,-0.2817450204 \backslash \mathrm{C},-1.1049847124,1.297642367,0.3 \quad 667336858 \backslash \mathrm{H},-1.8891555802,1$. $7958436817,-0.2077172175 \backslash \mathrm{H},-0.1901273171,1$.8946659609,0.355900396\H,-1.4404996418,1. $1476571177,1.3961531762 \backslash \mathrm{H},-0 \quad .4576178313,0.1408766737,-1.3203303779 \backslash \mathrm{O},-1.8850069551,-$ $0.8412594839,-0.3243934503 \backslash \mathrm{N}, 0.3350077875,-0.7492900303,0.3979802995 \backslash \mathrm{H}, 0.1128344903$, $-1.6074352267,0.8872238864 \backslash \mathrm{C}, 1.6291513118,-0.3833308105,0.2008315493 \backslash \mathrm{O}, 1.973667161$ $4,0.5807506358,-0.4666579972 \backslash \mathrm{H}, 2.349772065,-1.0471563155,0 \quad .7163983318 \backslash \backslash$ Version=DECAXP-OSF/1-G98RevA.11.3 \HF=-323.0674922 $\backslash \mathrm{S} 2=0.7$ 53606 $\backslash \mathrm{S} 2-1=0 . \backslash \mathrm{S} 2 \mathrm{~A}=0.750009 \backslash \mathrm{RMSD}=2$ $.047 \mathrm{e}-09 \backslash \mathrm{RMSF}=1.989 \mathrm{e}-06 \backslash \mathrm{Dipole}=-0.258 \quad 0082,-0.4264199,0.6200963 \backslash \mathrm{PG}=\mathrm{C} 01[\mathrm{X}(\mathrm{C} 3 \mathrm{H} 6 \mathrm{~N} 1 \mathrm{O}$ 2)]\\@

\section{$\mathrm{CHONH}-\mathrm{CHOCH}_{3}$ (TS)}

$1 \backslash 1 \backslash G I N C-S C 93 \backslash F T S \backslash U B 3 L Y P \backslash 6-31 G(d) \backslash C 3 H 6 N 1 O 2(2) \backslash G P W 501 \backslash 31-J a n-2003 \backslash 0 \backslash \backslash \#$ B3LYP/6-3 $1 \mathrm{G}(\mathrm{D})$ OPT $=($ NOEIGENTEST,TS,TIGHT,MAXCYC $=100)$ MAXDISK=134217728 FREQ IOP( $1 / 8=10) \backslash$ ts $\backslash \backslash 0,2 \backslash \mathrm{O},-2.0978595135,0.0122296666,-0.8502832152 \backslash \mathrm{C},-1.7899389214,-0.155466783$ $8,0.3135489926 \backslash \mathrm{N},-0.5294462564,-0.5269942799,0.7547586777 \backslash \mathrm{C}, 1.011197353,0.2603935849$, $-0.3810972655 \backslash \mathrm{H}, 0.4077702018,0.1551582147,-1.2994441469 \backslash \mathrm{C}, 2.1146620279,-0.7554372938$, $-0.2037567858 \backslash \mathrm{H}, 2.9303013337,-0.4906934995,-0.8907240697 \backslash \mathrm{H}, 1.7706076808,-1.761838578$ $9,-0.4558898225 \backslash \mathrm{H}, 2.5042150477,-0.7287842239,0.8172657529 \backslash \mathrm{O}, 0.968588673,1.3254445098$, $0.2681469929 \backslash \mathrm{H},-0.3447761383,-0.1758131584,1.7007331217 \backslash \mathrm{H},-2.5433503639,-0.10739925,1$ $.1296685517 \backslash \backslash$ Version=DEC-AXP-OSF $/ 1-G 98$ RevA.11 $3 \backslash \mathrm{HF}=-323.028956 \backslash \mathrm{S} 2=0.771675 \backslash \mathrm{S} 2-1=$ $0 . \mathrm{S} 2 \mathrm{~A}=0.750253 \backslash \mathrm{RMSD}=9.416 \mathrm{e}-09 \backslash \mathrm{RMSF}=2.783 \mathrm{e}-06 \backslash \mathrm{Dipole}=0.3370168,-0.6769992,0.718463$ $4 \backslash \mathrm{PG}=\mathrm{C} 01[\mathrm{X}(\mathrm{C} 3 \mathrm{H} 6 \mathrm{~N} 1 \mathrm{O} 2)] \backslash \backslash @$

\section{CHONH}

$1 \backslash 1 \backslash$ GINC-SC13\FOpt $\backslash U B 3 L Y P \backslash 6-31 G(d) \backslash C 1 H 2 N 1 O 1(2) \backslash G P W 501 \backslash 26-N o v-2002 \backslash 0 \backslash \ \#$ B3LYP/6 $-31 \mathrm{G}(\mathrm{D}) \mathrm{OPT}=(\mathrm{TIGHT}, \mathrm{MAXCYC}=100) \mathrm{MAXDISK}=104857600 \mathrm{SCF}=(\mathrm{TIGHT}, \mathrm{QC}, \mathrm{MAXCYC}$ $=100)$ INT(GRID=ULTRAFINE) FREQ $\backslash \backslash \mathrm{CH} 2 \mathrm{ON} *$ opt $\backslash \backslash 0,2 \backslash \mathrm{H},-1.1725420978,0.1368175509,-1$. $0436280672 \backslash \mathrm{N},-1.1776135672,0.1102778886,-0.0129801233 \backslash \mathrm{C}, 0.1219270194,0.108867096,0.4$ $01129902 \backslash \mathrm{H}, 0.2795167255,0.3106243875,1$. 4704585245\O,1.0505947783,-0.2340737168,-0.34 $28436258 \backslash \backslash$ Version=DEC-AXP $-\mathrm{OSF} / 1-\mathrm{G} 98 \mathrm{RevA} .11 .3 \backslash \mathrm{HF}=-169.2051116 \backslash \mathrm{S} 2=0.773683 \backslash \mathrm{S} 2-1=0$ $. \mathrm{S} 2 \mathrm{~A}=0.750093 \backslash \mathrm{R} \quad \mathrm{MSD}=0.000 \mathrm{e}+00 \backslash \mathrm{RMSF}=4.762 \mathrm{e}-06 \backslash \mathrm{Dipole}=-0.2081749,0.3204084,0.042875$ $4 \backslash \mathrm{PG}=\mathrm{C} 01[\mathrm{X}(\mathrm{C} 1 \mathrm{H} 2 \mathrm{~N} 1 \mathrm{O} 1)] \backslash \backslash @$ 


\section{$\mathrm{NH}_{2} \mathrm{COCH}_{3} \mathrm{CONH}_{2}$}

$1 \backslash 1 \backslash$ GINC-SC85\FOpt $\backslash U B 3 L Y P \backslash 6-31 G(d) \backslash C 3 H 7 N 2 O 2(2) \backslash G P W 501 \backslash 09-J a n-2003 \backslash 0 \backslash \backslash \#$ B3LYP/6-3 $1 \mathrm{G}^{*} \mathrm{OPT}=(\mathrm{TIGHT}, \mathrm{MAXCYC}=100)$ FREQ MAXDISK $=134217728 \backslash \backslash 1 \backslash \backslash 0,2 \backslash \mathrm{H},-1.8695500354,-0$ $.7817220018,-0.8230722432 \backslash \mathrm{N},-1.7795891424,-0.7218524773,0.1824571168 \backslash \mathrm{C},-0.5605820531$, $-0.404019728,0.6982068829 \backslash \mathrm{O},-0.2507148724,-0.5050376996,1.8738774641 \backslash \mathrm{C}, 0.4113576767,0$ $.1677989235,-0.3798809867 \backslash \mathrm{O}, 0.0152240931,-0.1053282118,-1.6639689335 \backslash \mathrm{C}, 1.8578880108,-$ $0.200723231,-0.0727030659 \backslash \mathrm{H}, 1.9918772365,-1.2813634369,-0.1816148031 \backslash \mathrm{H}, 2.1112036277$, $0.0836442587,0.9501923195 \backslash \mathrm{H}, 2.5258515948,0.3006761832,-0.7808173693 \backslash \mathrm{N}, 0.2410963973,1$ $.6402413277,-0.3852420932 \backslash \mathrm{H}, 0.8227354881,2.0512761621,-1.116042453 \backslash \mathrm{H},-0.7317329062,1$. $8906475441,-0.5611406733 \backslash \mathrm{H},-2.4489913613,-1.1872891571,0.7789848309 \backslash \backslash$ Version=DEC-A $\mathrm{XP}-\mathrm{OSF} / 1-\mathrm{G} 98 \mathrm{RevA} .11 .3 \backslash \mathrm{HF}=-378.429716 \backslash \mathrm{S} 2=0.755456 \backslash \mathrm{S} 2-1=0 . \backslash \mathrm{S} 2 \mathrm{~A}=0.750015 \backslash \mathrm{RMSD}=3.71$

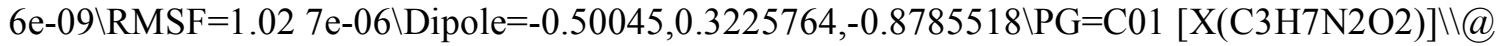

\section{$\mathrm{NH}_{2}-\mathrm{COCH}_{3} \mathrm{CONH}_{2}$ (TS)}

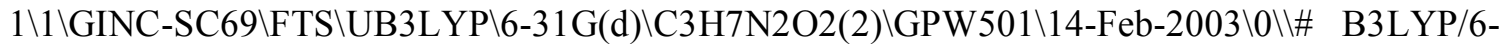
$31 \mathrm{G}$ (D) $\mathrm{OPT}=($ NOEIGENTEST,TS,TIGHT,MAXCYC=100) MAXDISK=134217728 FREQ IO $\mathrm{P}(1 / 8=10) \backslash \backslash \mathrm{ts} \backslash \backslash 0,2 \backslash \mathrm{H}, 1.3269363231,0.4110967259,2.2064947568 \backslash \mathrm{N}, 0.5257199583,-0.13911110$ $02,1.865710518 \backslash \mathrm{C}, 0.5727318361,0.2701374446,-0 . \quad 1388718267 \backslash \mathrm{O}, 0.6731229404,1.501044049$ $2,0.0506407467 \backslash \mathrm{H},-0.300441662,0.3 \quad 755857678,2.2008378354 \backslash \mathrm{O},-0.9895544214,-1.448189442$ $9,-0.8737163258 \backslash \mathrm{C},-\quad 0.8327197064,-0.3175844575,-0.4446973798 \backslash \mathrm{N},-1.8273427809,0.5758948$ $256,-0.1835287972 \backslash \mathrm{H},-1.5601884887,1.5440828162,-0.0594604214 \backslash \mathrm{H},-2.7616764039,0.3566$ $628452,-0.49889406 \backslash \mathrm{C}, 1.7387522734,-0.5873015204,-0.5723881564 \backslash \mathrm{H}, 1.8341081153,-0.5067$ $241627,-1.6627144833 \backslash \mathrm{H}, 1.5703222262,-1.6347645731,-0.3229088819 \backslash \mathrm{H}, 2.6611650777,-0.21$ $77711483,-0.1182779809 \backslash \backslash$ Version=D EC-AXP-OSF/1-G98RevA.11.3\HF=-378.4083726 $\backslash \mathrm{S} 2=0$. $765934 \backslash \mathrm{S} 2-1=0 . \mathrm{S} 2 \mathrm{~A}=0.75$ 0076 $\backslash \mathrm{RMSD}=8.990 \mathrm{e}-09 \backslash \mathrm{RMSF}=2.617 \mathrm{e}-06 \backslash \mathrm{Dipole}=-0.190961,0.8532$ 751,0.558484 9\PG=C01 [X(C3H7N2O2)]\\@

\section{$\mathrm{CH}_{3} \mathrm{COCONH}_{2}$}

$1 \backslash 1 \backslash$ GINC-SC49\FOpt $\backslash R B 3 L Y P \backslash 6-31 G(d) \backslash C 3 H 5 N 1 O 2 \backslash G P W 501 \backslash 09-J a n-2003 \backslash 0 \backslash \backslash \#$ B3 LYP/6-31 $\mathrm{G}^{*}$ FREQ OPT $=($ TIGHT,MAXCYC $=100)$ MAXDISK $=134217728 \backslash \backslash \mathrm{comf} 1 \backslash \backslash 0,1 \backslash \mathrm{N},-1.30651585$ $57,-1.2766314656,0 . \mid \mathrm{C},-0.0414469223,-0.7962585344,0 . \backslash \mathrm{C}, 0.0000057631,0.7520885817,0 . \backslash \mathrm{O},-1$ $.0395246048,1.3858559159,0 .|\mathrm{C}, 1.3720596349,1.3692680831,0.| \mathrm{O}, 0.9832448136,-1.46422273$ $49,0 . \mathrm{H}, 1.2893651659,2.4573233734,0 . \backslash \mathrm{H}, 1.9354293183,1.0270726452,0.8753547612 \backslash \mathrm{H}, 1.9354$ $293183,1.0270726452,-0.8753547612 \backslash \mathrm{H},-2.0797929415,-0.6260182568,0 . \backslash \mathrm{H},-1.4682923946,-2$.

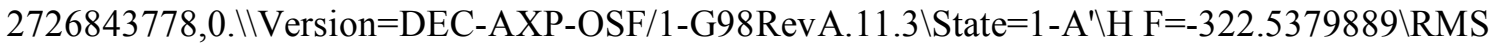
$\mathrm{D}=8.899 \mathrm{e}-09 \backslash \mathrm{RMSF}=2.974 \mathrm{e}-06 \backslash \mathrm{Dipole}=-0.4730716,-0.0542104,0 . \mid \mathrm{PG}=\mathrm{CS}[\mathrm{SG}(\mathrm{C} 3 \mathrm{H} 3 \mathrm{~N} 1 \mathrm{O} 2), \mathrm{X}($ H2)]\\,

\section{$\mathrm{CHONH}-\mathrm{COCH}_{3} \mathrm{CONH}_{2}(\mathrm{TS})$}

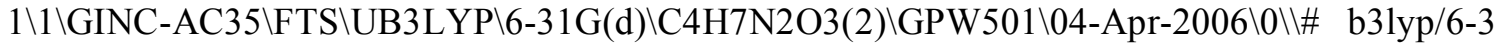
$1 \mathrm{G}(\mathrm{d}) \mathrm{opt}=(\mathrm{TS}$, noeigentest,calcfc) freq $\backslash \backslash$ comment $\backslash \backslash 0,2 \backslash \mathrm{N}, 0.3133055993,-0.2445065927,-0.4103$ $67274 \backslash \mathrm{C}, 2.5562211096,-0.2214508129,-0.5240 \quad 973874 \backslash \mathrm{H},-0.2390446469,0.3092654687,-1.070$ $2489778 \backslash \mathrm{C},-0.3515929507,-0.4976654685,0.7423832006 \backslash \mathrm{H},-1.4598553484,-0.5138644149,0.6$ $273563721 \backslash \mathrm{O}, 0 . \quad 1509520631,-0.7604834674,1.8341663945 \backslash \mathrm{O}, 3.0063995579,-1.0692777153,0.2$ $127281678 \backslash \mathrm{C}, 2.6494623285,-0.2522136351,-2.0293893843 \backslash \mathrm{H}, 2.0255832099,0 . \quad 5192987937,-2$ $.4807758403 \backslash \mathrm{H}, 2.3667280678,-1.2448983583,-2.3849126925 \backslash \mathrm{H}, 3.6931615565,-0.0635256842$, $-2.3078694949 \backslash \mathrm{O}, 2.357843437,2.2119101715,-0 \quad .6132218238 \backslash \mathrm{C}, 2.2986485846,1.2404221025,0$ 
$.1206891181 \backslash \mathrm{N}, 2.2358682728,1$. 2286984609,1.4617277084\H,1.9170067525,0.3683216433,1.9 $066044411 \backslash \mathrm{H}, 2.0 \quad 275824064,2.1061495085,1.9202674726 \backslash \backslash$ Version=IA64L-G03RevD.01 $\backslash$ State $=2-\mathrm{A} \backslash \mathrm{HF}=-491.7337401 \backslash \mathrm{S} 2=0.769116 \backslash \mathrm{S} 2-1=0 . \backslash \mathrm{S} 2 \mathrm{~A}=0.750174 \backslash \mathrm{RMSD}=6.224 \mathrm{e}-09 \backslash \mathrm{RMSF}=7.1$ 57e-06 $\backslash$ Thermal $=0 . \backslash$ Dipole $=-0.6086655,0.5919669,-0.6157646 \backslash \mathrm{PG}=\mathrm{C} 01[\mathrm{X}(\mathrm{C} 4 \mathrm{H} 7 \mathrm{~N} 2 \mathrm{O} 3)] \backslash \backslash @$

\section{$\mathrm{CH}_{3} \mathrm{CONH}-\mathrm{COCH}_{3} \mathrm{CONHCH}_{3}$ (TS)}

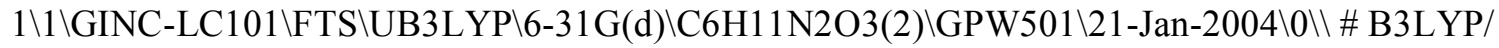
6-31G(D) OPT $=($ CALCFC,TIGHT,NOEIGENTEST,TS,MAXCYC $=100)$ MAXDISK $=1342177$ $28 \mathrm{SCF}=($ TIGHT,MAXCYC=100) FREQ $\|$ comment $\backslash \backslash 0,2 \backslash \mathrm{O},-0.9132414652,-1.0165191998,-1.66$ $82377451 \backslash \mathrm{C},-1.0589276154,-1.0153550912,-0.4593594697 \backslash \mathrm{N}, 0.8283811377,-0.9972542582,0.5$ $365335907 \backslash \mathrm{H}, 0.7297874109,-0.9421180762,1.554661321 \backslash \mathrm{C},-1.6787367141,-2.155264202,0.315$ $293409 \backslash \mathrm{H},-1.2074440404,-3.092463525,0.0128681549 \backslash \mathrm{H},-2.7431190293,-2.1989228932,0.0552$ $278886 \backslash \mathrm{H},-1.5925998964,-2.0058727053,1.3916259964 \backslash \mathrm{O},-1.6877666581,0.5268483573,1.354$ $5927566 \backslash \mathrm{C},-1.1706863786,0.4337729759,0.2497111418 \backslash \mathrm{N},-0.7845566244,1.4242600191,-0.563$ $1560082 \backslash \mathrm{H},-0.2725803047,1.1281056234,-1.3890667064 \backslash \mathrm{C},-0.7689234575,2.8204863655,-0.16$ $21925599 \backslash \mathrm{H},-1.2094703687,3.4429987795,-0.9472677277 \backslash \mathrm{H},-1.3585249572,2.9244764493,0.74$ $98176737 \backslash \mathrm{C}, 1.9133975537,-0.2891815254,0.0655955619 \backslash \mathrm{O}, 1.91750038,0.2492907515,-1.0384$ $378337 \backslash \mathrm{C}, 3.1378105774,-0.256498092,0.9793556778 \backslash \mathrm{H}, 4.0038568129,0.0594836809,0.39410$ $20633 \backslash \mathrm{H}, 3.3346045751,-1.2350948831,1.4295286612 \backslash \mathrm{H}, 2.9779348329,0.4620501,1.793174221$ $\backslash \mathrm{H}, 0.2552415254,3.1635952671,0.0279253896 \backslash \backslash$ Versi on $=x 86-$ Linux-G03RevB.03 $\backslash$ State $=2-\mathrm{A} \backslash \mathrm{H}$ $\mathrm{F}=-570.3689266 \backslash \mathrm{S} 2=0.770861 \backslash \mathrm{S} 2-1=0 . \backslash \mathrm{S} 2 \mathrm{~A}=0.75018 \backslash \mathrm{RMSD}=7.389 \mathrm{e}-09 \backslash \mathrm{RMSF}=2.641 \mathrm{e}-06 \backslash \mathrm{Dip}$ ole=0.3034447,0.3384088,0 .9771559 $\backslash \mathrm{PG}=\mathrm{C} 01$ [X(C6H11N2O3)]\\@,

\section{$\mathrm{CH}_{3} \mathrm{CONH}$}

$1 \backslash 1 \backslash$ GINC-LC23\FOpt $\backslash U B 3 L Y P \backslash 6-31 G(d) \backslash C 2 H 4 N 1 O 1(2) \backslash G P W 501 \backslash 21-J a n-2004 \backslash 0 \backslash \backslash \#$ B3LYP/6$31 \mathrm{G}(\mathrm{D}) \mathrm{OPT}=(\mathrm{TIGHT}, \mathrm{MAXCYC}=100) \mathrm{MAXDISK}=65536000 \mathrm{SCF}=(\mathrm{TIGHT}, \mathrm{MAX} \mathrm{CYC}=100)$ FREQ $\backslash \backslash$ comment $\backslash \backslash 0,2 \backslash O,-0.4488435686,1.2542323926,0.0464788303 \backslash \mathrm{C}, 0.0204011916,0.10744$ $7587,0.0388100162 \backslash \mathrm{C},-0.8238755161,-1.1374209931,-0.0446363578 \backslash \mathrm{H},-1.8637667782,-0.8988$ $996521,0.1864719345 \backslash \mathrm{N}, 1.379252535 \quad 7,-0.0395359198,-0.0680534743 \backslash \mathrm{H}, 1.8329135228,0.7763$ $915454,0.3628593253 \backslash \mathrm{H},-0.4490186589,-1.9000133396,0.6463147385 \backslash \mathrm{H},-0.7633013399,-1.55$ $47458187,-1.0561442711 \backslash \backslash$ Version=x86-Linux-G03RevB.03 $\backslash$ State=2-A $\backslash H F=-208.5321 \quad 14 \backslash \mathrm{S} 2=$ $0.767948 \backslash \mathrm{S} 2-1=0 . \backslash \mathrm{S} 2 \mathrm{~A}=0.75007 \backslash \mathrm{RMSD}=3.626 \mathrm{e}-09 \backslash \mathrm{RMSF}=3.076 \mathrm{e}-06 \backslash \mathrm{Dipol} \mathrm{e}=0.0115399,-0.54$ $32722,0.3092379 \backslash \mathrm{PG}=\mathrm{C} 01[\mathrm{X}(\mathrm{C} 2 \mathrm{H} 4 \mathrm{~N} 1 \mathrm{O} 1)] \backslash \backslash @$

\section{$\mathrm{CH}_{3} \mathrm{COCONHCH}_{3}$}

$1 \backslash 1 \backslash$ GINC-SC80\FOpt $\backslash R B 3 L Y P \backslash 6-31 G(d) \backslash C 4 H 7 N 1 O 2 \backslash G P W 501 \backslash 09-D e c-2003 \backslash 0 \backslash \backslash \#$ B3 LYP/6-31 $\mathrm{G}(\mathrm{D}) \mathrm{OPT}=(\mathrm{TIGHT}, \mathrm{MAXCYC}=100) \mathrm{MAXDISK}=52428800 \mathrm{SCF}=(\mathrm{TIGHT}, \mathrm{MAXCYC}=100) \mathrm{F}$ REQ $\mid$ lcomment $\backslash 0,1 \backslash C, 1.1496422175,-0.3480724959,0.1 O, 1.2118874349,-1.5647034288,0 . \backslash C, 2$. $3481715683,0.5611689826,0 . \mathrm{H}, 2.3162746603,1.2197681968,-0.8751552056 \backslash \mathrm{H}, 3.2645354539$,$0.0313517813,0 . \mathrm{H}, 2.3162746603,1.2197681968,0.8751552056 \backslash \mathrm{O},-0.3193686431,1.580162065$, $0 . \backslash \mathrm{C},-0.2279009883,0.3568119009,0 . \backslash \mathrm{N},-1.2539719466,-0.5241497171,0 . \backslash \mathrm{H},-0.9915651989,-1$. $5026149581,0 . \backslash \mathrm{C},-2.6467595008,-0.1246575705,0 . \backslash \mathrm{H},-3.1644127666,-0.4989938657,-0.890971$

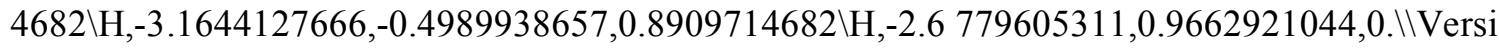
on=DEC-AXP-OSF $/ 1-G 03 R e v B .03 \backslash$ State $=1-A^{\prime} \backslash \mathrm{HF}=-361.8504386 \backslash \mathrm{RMSD}=6.019 \mathrm{e}-09 \backslash \mathrm{RMSF}=9$. 129e-07\Dipole=-0.368454,-0.376 5157,0.|PG=CS [SG(C4H3N1O2),X(H4)]\\@, 


\title{
TABLE S3: GAUSSIAN Archive Entries for B3-LYP/6-31G(d) Optimized Equilibrium Structures of Additional Species Involved in Reactions C1-C5
}

\author{
$\mathrm{CH}_{2} \mathrm{OCH}_{3}$ \\ $1 \backslash 1 \backslash$ GINC-SC36\FOpt $\backslash U B 3 L Y P \backslash 6-31 \mathrm{G}(\mathrm{d}) \backslash \mathrm{C} 2 \mathrm{H} 5 \mathrm{O} 1(2) \backslash \mathrm{GPW} 501 \backslash 20-A u g-2002 \backslash 0 \backslash \backslash \# \mathrm{~B} 3 \mathrm{LYP} / 6-31$ \\ $\mathrm{G}^{*} \mathrm{OPT}=$ TIGHT MAXDISK=91750400 $\backslash \mathrm{ch} 3-\mathrm{ch} 2-\operatorname{co}$ opt $\backslash \backslash 0,2 \backslash \mathrm{O},-0.6566945312,0 .,-1.074193663$ \\ $7 \backslash \mathrm{C},-0.5125909695,0 ., 0.2889643642 \backslash \mathrm{C}, 1.0158309568,0 ., 0.5703230577 \backslash \mathrm{H}, 1.1713364136,0 ., 1.65$ \\ $38806459 \backslash \mathrm{H}, 1.4802304219,0.8902997739,0.1390016341 \backslash \mathrm{H}, 1.4802304219,-0.8902997739,0.139$ \\ $0016341 \backslash \mathrm{H},-0.9488404656,0.9016022622,0.7529704322 \backslash \mathrm{H},-0.9488404656,-0.9016022622,0.752$ \\ $9704322 \backslash \backslash$ Version=DEC-AXP-OSF $/ 1-G 98 R$ RevA.11.3 $\backslash$ State $=2-A^{\prime} \backslash H F=-154.3689456 \backslash S 2=0.7529$ \\ $08 \backslash \mathrm{S} 2-1=0 . \backslash \mathrm{S} 2 \mathrm{~A}=0.750006 \backslash \mathrm{RMSD}=2.631 \mathrm{e}-09 \backslash \mathrm{RMSF}=5.111 \mathrm{e}-06 \backslash \mathrm{Dipole}=0.283268,0 ., 0.7747867$ \\ $\backslash \mathrm{PG}=\mathrm{CS}[\mathrm{SG}(\mathrm{C} 2 \mathrm{H} 1 \mathrm{O} 1), \mathrm{X}(\mathrm{H} 4)] \backslash \backslash @$ \\ $\mathrm{CH}_{2} \mathrm{O}-\mathrm{CH}_{3}$ (TS)

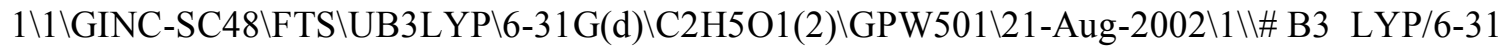 \\ $\mathrm{G}^{*} \mathrm{OPT}=(\mathrm{EF}, \mathrm{TS}, \mathrm{Z}-\mathrm{MATRIX}, \mathrm{TIGHT}) \mathrm{MAXDISK}=131072000 \mathrm{SCF}=(\mathrm{TIGHT}, \mathrm{QC}, \mathrm{MAXCYC}=$

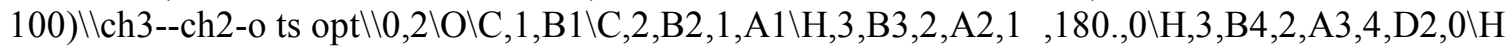

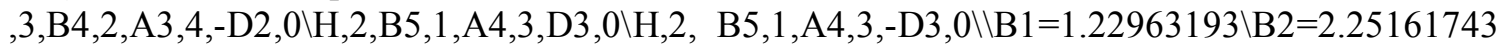 \\ $\backslash \mathrm{B} 3=1.08588009 \backslash \mathrm{B} 4=1.08392275 \backslash \mathrm{B} 5=1.10853465 \backslash \mathrm{A} 1=103.82991368 \backslash \mathrm{A} 2=99.81137277 \backslash \mathrm{A} 3=98$. \\ $14795922 \backslash \mathrm{A} 4=121.82926443 \backslash \mathrm{D} 2=-120.35334999 \backslash \mathrm{D} 3=97.94184213 \backslash \backslash$ Version=DEC-AXP-OSF $/$ \\ $1-G 98 R e v$ A.11.3 $\backslash$ State $=2-A^{\prime} \backslash H F=-154.3364528 \backslash \mathrm{S} 2=0.767097 \backslash \mathrm{S} 2-1=0 . \backslash \mathrm{S} 2 \mathrm{~A}=0.750085 \backslash \mathrm{RMS} \quad \mathrm{D}$ \\ $=0.000 \mathrm{e}+00 \backslash \mathrm{RMSF}=8.384 \mathrm{e}-07 \backslash$ Dipole $=0.4393826,0 ., 1.0017435 \backslash \mathrm{PG}=\mathrm{CS}[\mathrm{SG}(\mathrm{C} 2 \mathrm{H} 1 \mathrm{O} 1), \mathrm{X}(\mathrm{H} 4)] \backslash$ \\ $1 @$ \\ $\mathrm{CH}_{3}$ \\ $1 \backslash 1 \backslash$ GINC-SC118\FOpt $\backslash U B 3 L Y P \backslash 6-31 G(d) \backslash C 1 H 3(2) \backslash G P W 501 \backslash 11-S e p-2002 \backslash 0 \backslash \backslash \#$ B3 LYP/6-31G \\ * OPT=TIGHT MAXDISK=1342177280 GEOM=CHECK GUESS=READ $\backslash \backslash$ me opt $\backslash \backslash 0,2 \backslash \mathrm{C}, 0 ., 0$, \\ $0 . \backslash \mathrm{H}, 0 ., 0 ., 1.0827523064 \backslash \mathrm{H}, 0.9376910033,0 .,-0.5413761532 \backslash \mathrm{H},-0.9376910033,0 .,-0.5413761532$ \\ ॥Version=DEC-AXP-OSF $/ 1-G 98 R e v A .11 .3 \backslash \mathrm{HF}=-39.8382922 \backslash \mathrm{S} 2=0.753765 \backslash \mathrm{S} 2-1=0 . \mathrm{S} 2 \mathrm{~A}=0.75$ \\ $0007 \backslash \mathrm{RMSD}=9.540 \mathrm{e}-09 \backslash \mathrm{RMSF}=1.73$ 7e-08\Dipole=0.,0.,0.\PG=D03H [O(C1),3C2(H1)]\\@ \\ $\mathrm{CHOCONH}_{2}-\mathrm{CH}_{3}$ (TS)

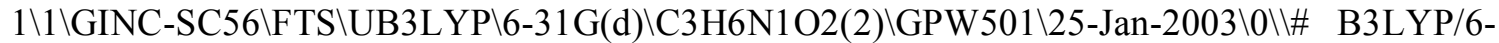 \\ $31 \mathrm{G}(\mathrm{D}) \mathrm{OPT}=(\mathrm{TS}, \mathrm{NOEIGENTEST,MAXCYC}=100$, TIGHT) $\mathrm{MAXDISK}=134217728$ FREQ $\backslash \backslash$ ts $\backslash$ \\ $\backslash 0,2 \backslash \mathrm{H},-1.9796816575,0.8162759644,-1.0237343158 \backslash \mathrm{C},-1.9823162 \quad 861,0.7985907484,0.060405$ \\ $828 \backslash \mathrm{C}, 0.2718440162,0.822049129,0.3357058344 \backslash \mathrm{O}, 0.441845128,0.8032121496,1.5567757557 \backslash$ \\ $\mathrm{H},-2.1973580788,-0.1405728587,0 \quad .5575997569 \backslash \mathrm{H},-2.2034310706,1.7140610779,0.5972879464$ \\ $\backslash \mathrm{C}, 0.4713513974,-\quad 0.4336047419,-0.5297665801 \backslash \mathrm{O}, 0.4528214646,-0.3733383545,-1.75095569$ \\ $32 \backslash \mathrm{H}, 0.2698070848,1.7566585905,-0.2546332592 \backslash \mathrm{N}, 0.6665614497,-1.5502224365,0.2150671$ \\ $329 \backslash \mathrm{H}, 0.9270186182,-2.4131991815,-0.239339541 \backslash \mathrm{H}, 0.7951074504,-1.4428677109,1.2127184$ \\ $889 \backslash \backslash$ Version=DEC-AXP-OSF $/ 1-G 98 R$ RevA.11.3\HF=-32 3.0461806 $\backslash \mathrm{S} 2=0.765883 \backslash \mathrm{S} 2-1=0 . \backslash \mathrm{S} 2 \mathrm{~A}$ \\ $=0.750082 \backslash \mathrm{RMSD}=6.950 \mathrm{e}-09 \backslash \mathrm{RMSF}=1.403 \mathrm{e}-06 \backslash \mathrm{Dipole}=-0.39878,-0.4758749,0.423199 \backslash \mathrm{PG}=\mathrm{C}$ \\ 01 [X(C3H6N1O2)]\@
}

$\mathrm{CHOCONH}_{2}$ 
$1 \backslash 1 \backslash G I N C-S C 112 \backslash F O p t \backslash R B 3 L Y P \backslash 6-31 G(d) \backslash C 2 H 3 N 1 O 2 \backslash G P W 501 \backslash 06-J a n-2003 \backslash 0 \backslash \backslash \#$ B 3LYP/6-3 $1 \mathrm{G}^{*} \mathrm{OPT}=(\mathrm{TIGHT}, \mathrm{MAXCYC}=100) \mathrm{MAXDISK}=134217728 \mathrm{FREQ} \|$ comf $1 \backslash \backslash 0,1 \backslash \mathrm{N},-0.4701623$ $416,0 .,-1.321657994 \backslash \mathrm{C},-0.5674360807,0 ., 0.0282626426 \backslash \mathrm{C}, 0.7980453726,0 ., 0.7263322416 \backslash \mathrm{O}, 1$. $8505057615,0 ., 0.1275975193 \backslash \mathrm{H}, 0.7231371608,0 ., 1.8308525991 \backslash \mathrm{O},-1.5949815851,0 ., 0.6896009$ $351 \backslash \mathrm{H}, 0.4437893605,0 .,-1.7534181824 \backslash \mathrm{H},-1.3036392928,0 .,-1.8909853997 \backslash \backslash$ Version=DEC-AX P-OSF/1-G9 8RevA.11.3 \State=1-A' $\backslash H F=-283.208857 \backslash R M S D=9.409 e-09 \backslash R M S F=6.053 e-06 \backslash D i p$ ole=0.1445741,0.,-0.821143\PG=CS [SG(C2H3N1O2)]1\@,

$\mathrm{CHONHCHO}-\mathrm{CH}_{3}$ (TS)

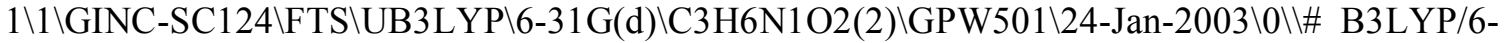
$31 \mathrm{G}(\mathrm{D}) \mathrm{OPT}=(\mathrm{TIGHT}, \mathrm{MAXCYC}=100$,TS,NOEIGENTEST) MAXDISK $=134217728 \mathrm{FREQ} \| \mathrm{ts}$ $\backslash \backslash 0,2 \backslash \mathrm{C}, 0.7789712252,0.3906843158,-0.3423058057 \backslash \mathrm{C}, 0.76713083 \quad 57,0.4114646872,1.7424851$ $246 \backslash \mathrm{H}, 1.8316364448,0.4073560539,1.9529552736 \backslash \mathrm{H}, 0.2197957311,-0.5090265697,1.9240008$ $46 \backslash \mathrm{H}, 0.2483947053,1.3461292031,1.9300976993 \backslash \mathrm{H}, 1.2431553574,-0.6049317237,-0.28685580$ $25 \backslash \mathrm{O}, 1.4074961904,1.4257146218,-0.6079189374 \backslash \mathrm{N},-0.6081777692,0.3083820043,-0.6637157$ $751 \backslash \mathrm{H},-1.0171276898,1.1568374067,-1.0393572837 \backslash \mathrm{C},-1.3897089576,-0.8031110482,-0.4756$ $865394 \backslash \mathrm{O},-1.0055345374,-1.8366931072,0.0409742622 \backslash \mathrm{H},-2.4226620089,-0.6614382463,-0.84$ $62295827 \backslash \backslash$ Version=DEC-AXP-OSF $/ 1-G 98 R e v A .11 .3 \backslash \mathrm{HF}=-323.0479747 \backslash \mathrm{S} 2=0.765582 \backslash \mathrm{S} 2-1=0$ $. \mathrm{S} 2 \mathrm{~A}=0.750068 \backslash \mathrm{RMSD}=5.541 \mathrm{e}-09 \backslash \mathrm{RMSF}=3 \quad .872 \mathrm{e}-06 \backslash \mathrm{Dipole}=-0.711261,0.4264629,0.1218761$ $\backslash \mathrm{PG}=\mathrm{C} 01[\mathrm{X}(\mathrm{C} 3 \mathrm{H} 6 \mathrm{~N} 1 \mathrm{O} 2)] \backslash \backslash @$

\section{CHONHCHO}

$1 \backslash 1 \backslash$ GINC-SC16 $\backslash F O p t \backslash R B 3 L Y P \backslash 6-31 G(d) \backslash C 2 H 3 N 1 O 2 \backslash G P W 501 \backslash 14-J u l-2003 \backslash 0 \backslash \backslash \#$ B3 LYP/6-31 $\mathrm{G}^{*} \mathrm{OPT}=(\mathrm{TIGHT}, \mathrm{MAXCYC}=100) \mathrm{MAXDISK}=77332480 \mathrm{FREQ} \backslash \backslash \mathrm{comf} 1 \backslash \backslash 0,1 \backslash \mathrm{H},-0.577976086$ $7,0 .,-2.2725529536 \backslash \mathrm{C},-0.7503573707,0 .,-1.1804492422 \backslash \mathrm{N}, 0.4416637698,0 .,-0.4672734224 \backslash \mathrm{C}, 0$. $5322888476,0 ., 0.9243916832 \backslash \mathrm{O}, 1.592622282,0 ., 1.5084513022 \backslash \mathrm{H},-0.453667778,0 ., 1.410530210$ $1 \backslash \mathrm{H}, 1.3232251569,0 .,-0.9703993934 \backslash \mathrm{O},-1.8519743497,0 .,-0.6784911211 \backslash \backslash$ Version=DEC-AXP-

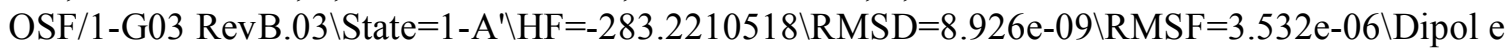
$=0.3563516,0 .,-1.0237815 \backslash \mathrm{PG}=\mathrm{CS}[\mathrm{SG}(\mathrm{C} 2 \mathrm{H} 3 \mathrm{~N} 1 \mathrm{O} 2)] \backslash @$

$\mathrm{CHONHCOCONH}_{2}-\mathrm{CH}_{3}$ (TS)

$1 \backslash 1 \backslash$ GINC-SC88\FTS \UB3LYP $\backslash 6-31 G(d) \backslash C 4 H 7 N 2 O 3(2) \backslash G P W 501 \backslash 03-D e c-2003 \backslash 0 \backslash \backslash \# \quad$ B3LYP/6$31 \mathrm{G}(\mathrm{D}) \mathrm{OPT}=(\mathrm{TIGHT}, \mathrm{MAXCYC}=100$,TS,NOEIGENTEST,CALCFC) $\mathrm{MAXDISK}=52428800$ FREQ SCF $=($ TIGHT,MAXCYC $=100) \backslash \backslash$ comment $\backslash \backslash 0,2 \backslash \mathrm{H},-2.0887704816,-0.1792522875,-1.0945$ $989391 \backslash \mathrm{C},-2.0851012286,-0.1658340021,-0.0084912824 \backslash \mathrm{C},-\quad 0.0763078825,-0.165895516,0.407$ $5337232 \backslash \mathrm{O},-0.0984757199,-0.1959412042,1 \quad .6581374479 \backslash \mathrm{H},-2.4316595872,-1.0667619763,0.4$ $875438939 \backslash \mathrm{H},-2.3597501855,0.7710824488,0.4655413436 \backslash \mathrm{C}, 0.1787391714,-1.4698718339,-0$. $4115853173 \backslash \mathrm{N}, 0.3682242396,0.9590970841,-0.3140777977 \backslash \mathrm{O}, 0.983652078,3.1574608567,-0$. $4129707664 \backslash \mathrm{C}, 0.6063100675,2.2021464073,0.2356120646 \backslash \mathrm{H}, 0.5307783644,0 \quad .8290433421,-1$. $3087793354 \backslash \mathrm{H}, 0.4228061237,2.2236053658,1.3212433134 \backslash \mathrm{H}, 0 \quad .093307529,-2.4408886136,1.3$ $749189837 \backslash \mathrm{N}, 0.1449733835,-2.5670149471,0.3 \quad 724640471 \backslash \mathrm{O}, 0.3800427687,-1.4387011122,-1$. $6166260941 \backslash \mathrm{H}, 0.3773110945,-3.4672218924,-0.0213128334 \backslash \backslash$ Version=DEC-AXP-OSF/1-G03 RevB.03 $\backslash$ State $=2-\mathrm{A} \backslash \mathrm{HF}=-491.7550463 \backslash \mathrm{S} 2=0.76429 \backslash \mathrm{S} 2-1=0 . \backslash \mathrm{S} 2 \mathrm{~A}=0.750067 \backslash \mathrm{RMSD}=8.793 \mathrm{e}-09 \backslash$ $\mathrm{RMSF}=3$. 149e-06\Dipole $=-0.8424893,-1.5952225,0.5047649 \backslash \mathrm{PG}=\mathrm{C} 01[\mathrm{X}(\mathrm{C} 4 \mathrm{H} 7 \mathrm{~N} 2 \mathrm{O} 3)] \backslash \backslash @$ 


\section{$\mathrm{CHONHCOCONH}_{2}$}

$1 \backslash 1 \backslash$ GINC-SC20\FOpt $\backslash$ RB3LYP $\backslash 6-31 G(d) \backslash C 3 H 4 N 2 O 3 \backslash G P W 501 \backslash 14-N o v-2002 \backslash 0 \backslash \ \#$ B3 LYP/6-31 $\mathrm{G}^{*} \mathrm{OPT}=(\mathrm{TIGHT}, \mathrm{MAXCYC}=100) \mathrm{MAXDISK}=134217728 \backslash \backslash \mathrm{comf} 1 \backslash \backslash 0,1 \backslash \mathrm{H}, 2.2170167165,0 .,-1$. $3417392542 \backslash \mathrm{C}, 2.2095946707,0 .,-0.2413449026 \backslash \mathrm{O}, 3.1980370017,0 ., 0.4556281003 \backslash \mathrm{N}, 0.9178026$ $809,0 ., 0.2814278934 \backslash \mathrm{C},-0.2297291284,0 .,-0.4732811447 \backslash \mathrm{O},-0.2827192494,0 .,-1.691983722 \backslash \mathrm{C},-$ $1.4922304395,0 ., 0.4174727972 \backslash \mathrm{O},-1.4039511429,0 ., 1.6387003671 \backslash \mathrm{N},-2.6241491426,0 .,-0.3119$ $462753 \backslash \mathrm{H},-3.5224153971,0 ., 0.1486476179 \backslash \mathrm{H},-2.5602182726,0 .,-1.3211147338 \backslash \mathrm{H}, 0.793298692$ 7,0.,1.2919965807\\Version=DEC-AXP-OSF $/ 1-G 98 R$ RevA.11.3 \State $=1-A^{\prime} \backslash H F=-451.9321664 \backslash \mathrm{R}$ $\mathrm{MSD}=4.580 \mathrm{e}-09 \backslash \mathrm{RMSF}=3.186 \mathrm{e}-06 \backslash \mathrm{Dipole}=-1.4527224,0 .,-0.5664538 \backslash \mathrm{PG}=\mathrm{CS}[\mathrm{SG}(\mathrm{C} 3 \mathrm{H} 4 \mathrm{~N} 2 \mathrm{O} 3)$ ]1@

\section{$\mathrm{CH}_{3} \mathrm{CONHCOCONHCH}-\mathrm{CH}_{3}$ (TS)}

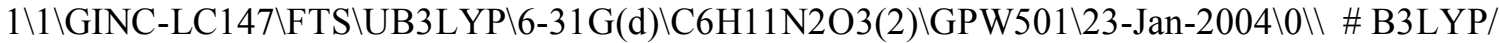
6-31G(D) OPT $=($ CALCFC,TS,NOEIGENTEST,TIGHT,MAXCYC $=100)$ MAXDISK $=5242880$ $0 \mathrm{SCF}=($ TIGHT,MAXCYC=100) FREQ $\backslash \backslash$ comment $\backslash \backslash 0,2 \backslash \mathrm{H},-0.9013929881,-1.9751949715,1.583$ $8901726 \backslash \mathrm{C},-0.0481430923,-1.3063620681,1.6291779633 \backslash \mathrm{C},-0.067930427,-0.3823430689,-0.22$ $62665682 \backslash \mathrm{O},-0.1095485754,-1.393934151,-0.9550080971 \backslash \mathrm{O},-1.3274649713,1.5380744663,0.5$ $878866229 \backslash \mathrm{C},-1.3668432267,0.4352297658,0.0460644733 \backslash \mathrm{N}, 1.0466021985,0.4796142145,-0.1$ $167368408 \backslash \mathrm{H}, 0.7977562228,1.4081705201,0.211612363 \backslash \mathrm{C}, 2.385354471,0.110110643,-0.175$ $3712955 \backslash \mathrm{O}, 2.7539652256,-1.0449771718,-0.2766000175 \backslash \mathrm{C}, 3.3445332843,1.2896546394,-0.11$ $395983 \backslash \mathrm{H}, 3.0753141084,1.9974570125,0.6784985198 \backslash \mathrm{H}, 3.328 \quad 8681719,1.8341219494,-1.0656$ $313441 \backslash \mathrm{H}, 4.3530750395,0.9125534784,0.0607924207 \backslash \mathrm{N},-2.4643957172,-0.2055502107,-0.395$ $1352375 \backslash \mathrm{H},-2.28538936,-1.078 \quad 1693652,-0.8799012608 \backslash \mathrm{C},-3.80391565,0.342739805,-0.302661$ $6668 \backslash \mathrm{H},-3.740156333,1.2926641742,0.2306026109 \backslash \mathrm{H},-4.46635383,-0.335655169,0.24662254$ $81 \backslash \mathrm{H},-4.2266261318,0.5195763345,-1.2983519965 \backslash \mathrm{H},-0.1334748376,-0.4561176016,2.299865$ $3127 \backslash \mathrm{H}, 0.9289889825,-1.7653378331,1.522978679 \backslash \backslash$ Version=x $86-$ Linux-G03RevB.03 $\backslash$ State $=2-$ $\mathrm{A} \backslash \mathrm{HF}=-570.3861539 \backslash \mathrm{S} 2=0.766458 \backslash \mathrm{S} 2-1=0 . \backslash \mathrm{S} 2 \mathrm{~A}=0.750075 \backslash \mathrm{RMSD}=8.859 \mathrm{e}-09 \backslash \mathrm{RMSF}=1.316 \mathrm{e}-0$ 6\Dipole=-0.9759682,0.6449513,0.4 41587\PG=C01 [X(C6H11N2O3)]\@

\section{$\mathrm{CH}_{3} \mathrm{CONHCOCONHCH}$}

$1 \backslash 1 \backslash G I N C-S C 160 \backslash F O p t \backslash R B 3 L Y P \backslash 6-31 G(d) \backslash C 5 H 8 N 2 O 3 \backslash G P W 501 \backslash 11-J a n-2004 \backslash 0 \backslash \backslash \#$ B 3LYP/6-3 $1 \mathrm{G}(\mathrm{D}) \mathrm{OPT}=(\mathrm{TIGHT}, \mathrm{MAXCYC}=100) \mathrm{MAXDISK}=65536000 \mathrm{SCF}=($ TIGHT,MAXCY C=100 $) \mathrm{F}$ REQ \|commnet $\backslash 0,1 \backslash \mathrm{N},-0.9703323798,-0.4742269473,0 . \backslash \mathrm{C},-0.0040698936,0.5059108601,0 . \mid \mathrm{C}, 1$. $4047321313,-0.1401535303,0.1 \mathrm{O}, 1.5460087377,-1.3628981712,0.1 \mathrm{O},-0.1591554194,1.71184243$ $55,0.1 \mathrm{O},-2.9074152123,0.7723086068,0 . \backslash \mathrm{C},-2.3689411505,-0.3105959155,0 . \mid \mathrm{C},-3.1151508296,-$ $1.633974641,0 . \backslash \mathrm{H},-4.1865679083,-1.4314291473,0 . \backslash \mathrm{H},-2.8578762322,-2.2277042776,-0.88532$ $42603 \backslash \mathrm{H},-2.8578762322,-2.2277042776,0.8853242603 \backslash \mathrm{N}, 2.3792810319,0.7852552912,0 . \backslash \mathrm{H}, 2.0$ $616644703,1.7483756753,0 . \mathrm{CC}, 3.7929863343,0.4616060027,0 . \backslash \mathrm{H}, 3.8862886626,-0.6255236279$ $, 0 . \backslash \mathrm{H}, 4.2860791109,0.8657868998,0.8911472665 \backslash \mathrm{H}, 4.2860791109,0.8657868998,-0.891147266$ $5 \backslash \mathrm{H},-0.5732759467,-1.411566177,0 . \mid \backslash$ Version=DEC-AXP-OSF $/ 1-G 03 R e v B .03 \backslash$ State $=1-A^{\prime} \backslash \mathrm{HF}=-$ $530.5631101 \backslash \mathrm{RMSD}=5.767 \mathrm{e}-09 \backslash \mathrm{RMSF}=1.351 \mathrm{e}-06 \backslash \mathrm{Dipole}=0.9764798,-0.7862153,0 . \backslash \mathrm{PG}=\mathrm{C} \mathrm{S}[\mathrm{S}$ G(C5H4N2O3),X(H4)]\\@ 


\title{
TABLE S4: GAUSSIAN Archive Entries for B3-LYP/6-31G(d) Optimized Equilibrium Structures of Additional Species Involved in Reactions of the Leucine Residue
}

\author{
Reactant $\mathrm{CH}_{3} \mathrm{CONHCO}\left(\mathrm{CH}_{2} \mathrm{CH}\left(\mathrm{CH}_{3}\right)_{2}\right) \mathrm{CONHCH}_{3}$
}

$1 \backslash 1 \backslash$ GINC-AC1 \FOpt $\backslash$ UB3LYP $\backslash 6-31 G(d) \backslash C 9 H 17 N 2 O 3(2) \backslash G P W 501 \backslash 16-F e b-2006 \backslash 0 \backslash \backslash \#$ b31yp/6-3 $1 \mathrm{G}(\mathrm{d})$ opt freq $\backslash \backslash$ comment $\backslash \backslash 0,2 \backslash \mathrm{H}, 0.98987,1.03832,-1.47796 \backslash \mathrm{C}, 0.06134,0.98421,-0.90409 \backslash \mathrm{C},-0.00$ $27,-0.54203,-0.48781 \backslash \mathrm{O},-0.06997,-1.28484,-1.5996 \backslash \mathrm{H},-0.76765,1.17277,-1.59501 \backslash \mathrm{C}, 0.07754,2.0$ $2282,0.23904 \backslash \mathrm{H}, 0.56805,1.56651,1.10861 \backslash \mathrm{C}, 0.92439,3.23324,-0.19328 \backslash \mathrm{H}, 0.50007,3.7123,-1.08$ $529 \backslash \mathrm{H}, 0.96141,3.98739,0.60171 \backslash \mathrm{H}, 1.95198,2.93576,-0.42977 \backslash \mathrm{C},-1.32683,2.48034,0.66451 \backslash \mathrm{H},-1$ $.86399,2.92923,-0.18142 \backslash \mathrm{H},-1.25891,3.24016,1.45203 \backslash \mathrm{H},-1.92979,1.65828,1.05946 \backslash \mathrm{N}, 1.0756,-0$ $.90282,0.42382 \backslash \mathrm{C},-1.35562,-0.80995,0.32014 \backslash \mathrm{O},-1.35313,-0.81159,1.54275 \backslash \mathrm{N},-2.41715,-1.0001$ $5,-0.4798 \backslash \mathrm{H},-2.21302,-1.05906,-1.47037 \backslash \mathrm{C},-3.76475,-1.22535,0.01689 \backslash \mathrm{H},-4.17254,-2.1524,-0.3$ $9907 \backslash \mathrm{H},-4.42707,-0.39392,-0.24959 \backslash \mathrm{H},-3.71724,-1.3099,1.10325 \backslash \mathrm{C}, 2.39778,-0.90521,0.06542 \backslash \mathrm{H}$ $, 0.79694,-1.14206,1.36637 \backslash \mathrm{O}, 2.7967,-0.54796,-1.0358 \backslash \mathrm{C}, 3.35016,-1.41435,1.1397 \backslash \mathrm{H}, 3.65091,-2$ $.43788,0.88898 \backslash \mathrm{H}, 2.91541,-1.41758,2.14428 \backslash \mathrm{H}, 4.2498,-0.79427,1.13388 \backslash \backslash$ Version=IA64L-G03 RevD.01 $\backslash \mathrm{Sta}$ te $=2-\mathrm{A} \backslash \mathrm{HF}=-688.343829 \backslash \mathrm{S} 2=0.754235 \backslash \mathrm{S} 2-1=0 . \backslash \mathrm{S} 2 \mathrm{~A}=0.750012 \backslash \mathrm{RMSD}=4.497 \mathrm{e}-09 \backslash$ $\mathrm{RMSF}=3.311 \mathrm{e}-06 \backslash$ Thermal $=0 . \mid$ Dipole $=-1.4093634,0.0504984,0.5561541 \backslash \mathrm{PG}=\mathrm{C} 01[\mathrm{X}(\mathrm{C} 9 \mathrm{H} 17 \mathrm{~N} 2$ O3)]\@

Transition Structure Pathway A $\mathrm{CH}_{3} \mathrm{CONHCO}\left(\mathrm{CH}_{2} \mathrm{CH}\left(\mathrm{CH}_{3}\right)_{2}\right)--\mathrm{CONHCH}_{3}$

$1 \backslash 1 \backslash$ GINC-AC23\FTS $\backslash$ UB3LYP $\backslash 6-31 G(d) \backslash C 9 H 17 N 2 O 3(2) \backslash G P W 501 \backslash 02-D e c-2005 \backslash 0 \backslash \$ B3LYP/ 6-31G(D) OPT=(TS,NOEIGENTEST,CALCFC,MAXCYC=100) FREQ $\backslash \backslash$ comment $\backslash \backslash 0,2 \backslash C,-1.24$ $62485333,-0.6237149472,0.189230476 \backslash \mathrm{C}, 0.236468633,0.02628519 \quad 17,-0.9061430558 \backslash \mathrm{O},-1.338$ $0455289,-0.2551988529,1.3328773501 \backslash \mathrm{N},-2.0143441783,-1.4013797689,-0.5580156503 \backslash \mathrm{H},-1.6$ $00111041,-1.6240825882,-1.4602335098 \backslash \mathrm{C},-3.1252108839,-2.1808833173,-0.0208246905 \backslash \mathrm{H},-3$ $.5517354641,-1.6503334795,0.8323348099 \backslash \mathrm{H},-3.8915585734,-2.2936690236,-0.7922563173 \backslash \mathrm{H}$ , -2.7898880989,-3.1720927912,0.3049577604\H,0.5742210044,1.9352279716,- 1.7695788683 $\backslash \mathrm{C},-0.0684649194,1.5462713346,-0.9671294633 \backslash 0,0.0416738926,-0.6419114404,-1.96287294$ $02 \backslash \mathrm{H},-1.0909638495,1.6189861493,-1.3505839418 \backslash \mathrm{C}, 0.1044085436,2.4036880099,0.31223856$ $37 \backslash \mathrm{H}, 0.02723651,1.7457982331,1.1860781205 \backslash \mathrm{C}, 1.4688741795,3.1159536712,0.347322198 \backslash \mathrm{H}$, $1.5489371572,3.8386950194,-0.4747750888 \backslash \mathrm{H}, 1.6042686457,3.6629983798,1.2873986661 \backslash \mathrm{H}$, $2.3177235163,2.4268251475,0.2434546818 \backslash \mathrm{C},-1.0295918523,3.4347538374,0.426 \quad 9682684 \backslash \mathrm{H}$ $,-1.0472693188,4.1019805267,-0.4446368721 \backslash \mathrm{H},-0.9088824964,4.0 \quad 573624056,1.3212590614 \backslash$ $\mathrm{H},-2.0041227158,2.9383446185,0.4928481723 \backslash \mathrm{N}, 1.3 \quad 701137468,-0.3009789539,-0.0739633469$ $\backslash \mathrm{C}, 1.6830033209,-1.5839996006,0.3 \quad 387214542 \backslash \mathrm{H}, 1.8166914039,0.4616442234,0.4149273031 \backslash$ $\mathrm{O}, 0.9983513432,-2.5 \quad 56308638,0.0669953815 \backslash \mathrm{C}, 2.9738006326,-1.6901030139,1.1387478366 \backslash$ H,2.92 35853262,-2.5796922675,1.7691310732\H,3.164120634,-0.8101169925,1.7630 33701\ H,3.8192879996,-1.8035200171,0.4497063741 \\Version=IA64L-G03RevC .02 $\backslash$ State=2-A $\backslash H F=-$ $688.3355785 \backslash \mathrm{S} 2=0.759932 \backslash \mathrm{S} 2-1=0 . \backslash \mathrm{S} 2 \mathrm{~A}=0.750035 \backslash \mathrm{RMSD}=8.783 \mathrm{e}-09 \backslash \mathrm{RMSF}=5.315 \mathrm{e}-06 \backslash \mathrm{Dipol}$ $\mathrm{e}=-0.2155432,0.7136984,0.4166054 \backslash \mathrm{PG}=\mathrm{C} 01[\mathrm{X}$ (C9H17N2O3)]\@

\section{$\mathrm{CH}_{3} \mathrm{CONHCO}\left(\mathrm{CH}_{2} \mathrm{CH}\left(\mathrm{CH}_{3}\right)_{2}\right)$}

$1 \backslash 1 \backslash$ GINC-AC38\FOpt $\backslash R B 3 L Y P \backslash 6-31 G(d) \backslash C 7 H 13 N 1 O 2 \backslash G P W 501 \backslash 01-D e c-2005 \backslash 0 \backslash \backslash \#$ B 3LYP/6-3 1G(D) OPT FREQ $\backslash \backslash$ comment $\backslash \backslash 0,1 \backslash \mathrm{H}, 0.8543873995,1.6000406652,-1.1102947611 \backslash \mathrm{C},-0.1688920$ $517,1.2363270969,-0.9426432972 \backslash \mathrm{C},-0.170636699,-0.2789828447,-0.7640475716 \backslash \mathrm{O},-1.041624$ 
$6129,-0.9990914845,-1.1999634631 \backslash \mathrm{H},-0.7486066411,1.447947351,-1.8466809643 \backslash \mathrm{C},-0.79202$ $91687,1.9888626189,0.2621634025 \backslash \mathrm{H},-0.2447961164,1.6865666627,1.1684552068 \backslash \mathrm{C},-0.61155$ $24513,3.5032960695,0.0865322246 \backslash \mathrm{H},-1.1264696086,3.8558118565,-0.8160396465 \backslash \mathrm{H},-1.0292$ $393546,4.0478387175,0.9411375655 \backslash \mathrm{H}, 0.4464537494,3.7780727643,-0.0026421913 \backslash \mathrm{C},-2.2713$ $066674,1.6256742076,0.4539747478 \backslash \mathrm{H},-2.8614778665,1.9390214538,-0.4162821032 \backslash \mathrm{H},-2.6805$ $081429,2.1307542241,1.3364543201 \backslash \mathrm{H},-2.4164823128,0.5483831263,0.5778095883 \backslash \mathrm{N}, 0.92069$ $35059,-0.7462176129,-0.007283057 \backslash \mathrm{C}, 1.1877578759,-2.0662597413,0.3997723442 \backslash \mathrm{H}, 1.591605$ $257,-0.0469469287,0.2840310199 \backslash \mathrm{O}, 0.4787356007,-3.0100291202,0.1369230896 \backslash \mathrm{C}, 2.4747884$ $44,-2.1828560076,1.2074268392 \backslash \mathrm{H}, 2.4476971015,-1.5384105356,2.0944453714 \backslash \mathrm{H}, 3.34285798$ $75,-1.8885802424,0.6048525276 \backslash \mathrm{H}, 2.5940604132,-3.2203793821,1.5209863176 \backslash \backslash$ Version=IA64 L-G03RevC.02 $\backslash$ State $=1-A \backslash H F=-479.8015289 \backslash$ RMSD $=7.286 \mathrm{e}-09 \backslash \mathrm{RMSF}=1.316 \mathrm{e}-05 \backslash$ Dipole $=1.42$ $81455,1.6805394,0.75$ 64674 $\backslash \mathrm{PG}=\mathrm{C} 01$ [X(C7H13N1O2)]\\@

Transition Structure Pathway B $\mathrm{CH}_{3} \mathrm{CONH}--\mathrm{CO}\left(\mathrm{CH}_{2} \mathrm{CH}\left(\mathrm{CH}_{3}\right)_{2}\right) \mathrm{CONHCH}_{3}$

$1 \backslash 1 \backslash$ GINC-AC37\FTS $\backslash U B 3 L Y P \backslash 6-31 G(d) \backslash C 9 H 17 N 2 O 3(2) \backslash G P W 501 \backslash 02-D e c-2005 \backslash 0 \backslash \backslash \#$ B3LYP/ 6-31G(D) OPT $=($ TS,CALCFC,NOEIGENTEST,MAXCYC=100) FREQ $\backslash \backslash$ comment $\backslash \backslash 0,2 \backslash \mathrm{N},-0.96$ $51319999,-0.8741900929,-0.585181808 \backslash \mathrm{C}, 0.3635801748,-0.22999 \quad 36926,0.7809003793 \backslash \mathrm{H},-0.6$ $107094286,-1.8217303391,-0.7509807961 \backslash \mathrm{C},-2.3398850918,-0.8878498858,-0.2887959518 \backslash \mathrm{O},-$ $2.8732123964,0.0107222125,0.3359490749 \backslash \mathrm{C},-3.1155654395,-2.0610450892,-0.8720810501 \backslash \mathrm{H}$

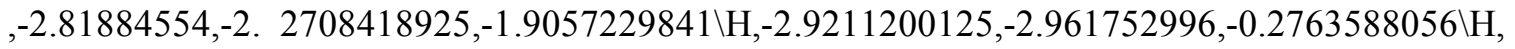
$-4.1822779826,-1.8333551173,-0.8361514314 \backslash \mathrm{C}, 0.0354579261,1.2308507058,1.0412523912 \backslash$ $\mathrm{H},-0.9134787051,1.2144990724,1.5821744399 \backslash \mathrm{H}, 0.8009977495,1.5493199421,1.766808245 \backslash \mathrm{C}$, $-0.0116818878,2.2377616328,-0.1446223535 \backslash \mathrm{H},-0.0059544624,1.665521998,-1.0777349076 \backslash \mathrm{C}$

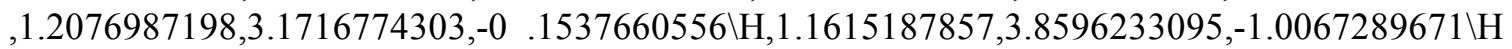

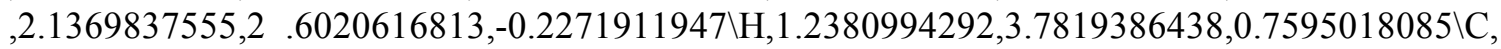
- $1.3134771445,3.0540305208,-0.0909299251 \backslash \mathrm{H},-2.1924364991,2.4027474952,-0.1061947504$ $\backslash \mathrm{H},-1.3583670537,3.6592442315,0.8247042098 \backslash \mathrm{C}, 1.6227187227,-0.5799516204,-0.072308317$ $5 \backslash \mathrm{O}, 2.2205809758,0.2351600702,-0.7604998491 \backslash \mathrm{N}, 1.9567467395,-1.8925755967,0.050866425$ $2 \backslash \mathrm{H}, 1.4672360794,-2.3886201908,0.787192519 \backslash \mathrm{C}, 3.1570807963,-2.4508841585,-0.543982933$ $2 \backslash \mathrm{H}, 3.5053231116,-1.7556364344,-1.3095644787 \backslash \mathrm{H}, 3.9530703133,-2.5826087963,0.19980914$ $73 \backslash \mathrm{H}, 2.9415780159,-3.4208585339,-1.0040732685 \backslash \mathrm{O},-0.0228281279,-1.1185802 \quad 919,1.59078$ $60608 \backslash \mathrm{H},-1.3748050026,3.7418167677,-0.9431605013 \backslash \backslash$ Version=I A64L-G03RevC.02 $\backslash$ State $=2-$ $\mathrm{A} \backslash \mathrm{HF}=-688.3039759 \backslash \mathrm{S} 2=0.770672 \backslash \mathrm{S} 2-1=0 . \backslash \mathrm{S} 2 \mathrm{~A}=0.75 \quad 0209 \backslash \mathrm{RMSD}=6.467 \mathrm{e}-09 \backslash \mathrm{RMSF}=6.859 \mathrm{e}-0$ 6\Dipole=0.1226612,-1.8214086,-0.4146 933 $\mathrm{PG}=\mathrm{C} 01$ [X(C9H17N2O3)]\\@

\section{$\mathrm{CO}\left(\mathrm{CH}_{2} \mathrm{CH}\left(\mathrm{CH}_{3}\right)_{2}\right) \mathrm{CONHCH}_{3}$}

$1 \backslash 1 \backslash$ GINC-AC43\FOpt $\backslash R B 3 L Y P \backslash 6-31 G(d) \backslash C 7 H 13 N 1 O 2 \backslash G P W 501 \backslash 01-D e c-2005 \backslash 0 \backslash \backslash \#$ B 3LYP/6-3 1G(D) OPT FREQ $\mid \backslash$ comment $\backslash \backslash 0,1 \backslash \mathrm{H}, 0.8845529905,1.5414634846,-1.6578212399 \backslash \mathrm{C}, 0.26914337$ $13,1.2154549894,-0.8121718649 \backslash \mathrm{C}, 0.2049360472,-0.2895873475,-0.8368052464 \backslash \mathrm{O}, 1.01362446$ $52,-1.0029471266,-1.4074096032 \backslash \mathrm{H},-0.7469477494,1.6071537159,-0.9265134494 \backslash \mathrm{C}, 0.8619034$ $545,1.765204526,0.5168845718 \backslash \mathrm{H}, 0.2680909917,1.3416250432,1.3366773548 \backslash \mathrm{C}, 2.3293239691$ ,1.35 31289201,0.6989832648\Н,2.9555962632,1.7769549619,-0.0964377131\H,2.71 5905986,1 $.7169684124,1.6580024253 \backslash \mathrm{H}, 2.4599636917,0.265886698,0.6752668068 \backslash \mathrm{C}, 0.7066672135,3.29$ $15173073,0.5596675101 \backslash \mathrm{H}, 1.2548782518,3.7667487381,-0.2640540219 \backslash \mathrm{H}, 1.1022265383,3.695$ $5162298,1.4989408366 \backslash \mathrm{H},-0.3452880323,3.5884723657,0.4822655556 \backslash \mathrm{C},-0.9652644873,-0.938$ $2318788,-0.0541937248 \backslash \mathrm{O},-1.8002809238,-0.2595975181,0.5372858509 \backslash \mathrm{N},-0.9322792886,-2.2$ $885399797,-0.1132891506 \backslash \mathrm{H},-0.1695901235,-2.6833290585,-0.6508263354 \backslash \mathrm{C},-1.913960622,-3$ 
$.139215669,0.5284375643 \backslash \mathrm{H},-1.4395540508,-3.8101375046,1.2540953361 \backslash \mathrm{H},-2.4544486423,-3$ $.7443896851,-0.2089483659 \backslash \mathrm{H},-2.6226731037,-2.4924214721,1.0485544332 \backslash \backslash$ Version=IA64LG03RevC.02 $\backslash$ State $=1-A \backslash H F=-479.7917765 \backslash$ RMSD $=4.765 \mathrm{e}-09 \backslash \mathrm{RMSF}=3.686 \mathrm{e}-06 \backslash$ Dipole $=0.0865$ $57,-0.4650125,0.0542682 \backslash \mathrm{PG}=\mathrm{C} 01[\mathrm{X}(\mathrm{C} 7 \mathrm{H} 13 \mathrm{~N} 1 \mathrm{O} 2)] \backslash \backslash$

Transition Structure Pathway $\mathrm{C} \mathrm{CH}_{3} \mathrm{CONHCO}--\left(\mathrm{CH}_{2} \mathrm{CH}\left(\mathrm{CH}_{3}\right)_{2}\right) \mathrm{CONHCH}_{3}$

$1 \backslash 1 \backslash$ GINC-AC11 $\backslash$ FTS $\backslash$ UB3LYP $\backslash 6-31 G(d) \backslash C 9 H 17 N 2 O 3(2) \backslash G P W 501 \backslash 02-D e c-2005 \backslash 0 \backslash \backslash \#$ B3LYP/ 6-31G(D) OPT=(TS,NOEIGENTEST,CALCFC,MAXCYC=100) FREQ $\backslash \backslash$ comment $\backslash \backslash 0,2 \backslash C, 0.021$ $4333311,1.1022665042,-1.0099915109 \backslash \mathrm{C},-0.0168922974,-0.85381 \quad 8841,-0.4766003362 \backslash \mathrm{H}, 0.97$ $47015348,0.9740003091,-1.5175447434 \backslash \mathrm{H},-0.8276 \quad 456097,1.12515013,-1.6913453441 \backslash \mathrm{C}, 0.001$ $9957109,2.0695296649,0.152237954 \backslash \mathrm{H}, 0.4038359188,1.5579878058,1.038246753 \backslash \mathrm{C},-1.40559$ $77326,2.5812982129,0.490731378 \backslash \mathrm{H},-1.3634604869,3.301707327,1.3155729865 \backslash \mathrm{H},-2.0666190$ $465,1.7670972308,0.798742652 \backslash \mathrm{H},-1.8544650508,3.0895960487,-0.3722122818 \backslash \mathrm{C}, 0.9586114$ $835,3.2439003938,-0.1639600272 \backslash \mathrm{H}, 1.9769633426,2.8887299055,-0 \quad .3549108902 \backslash \mathrm{H}, 0.6232497$ $05,3.8010778938,-1.0469989461 \backslash \mathrm{C},-1.3327884593,-0.850220575,0.3487495187 \backslash \mathrm{O},-1.3309533$ $617,-0.6326652877,1.5601849351 \backslash \mathrm{N},-2.4081552903,-1.1249761076,-0.4129931908 \backslash \mathrm{H},-2.19599$ $00477,-1.3681654578,-1.3745619885 \backslash \mathrm{C},-3.7546101687,-1.2441349986,0.112506657 \backslash \mathrm{H},-3.7229$ $100163,-0.9968001271,1.174912369 \backslash \mathrm{H},-4.4362384551,-0.554746215,-0.39834059 \quad 77 \backslash \mathrm{H},-4.133$ $6595117,-2.2660183553,-0.0043841156 \backslash \mathrm{O},-0.0246637854,-1.3572954903,-1.628000038 \backslash \mathrm{H}, 0.99$ $07040471,3.9420976674,0.6808126266 \backslash \mathrm{N}, 1.0821172021,-0.9705957094,0.4177262986 \backslash \mathrm{C}, 2.42$ $4525086,-0.913855651,0.0809263896 \backslash \mathrm{H}, 0.8091177829,-0.9586925004,1.3949179425 \backslash \mathrm{O}, 2.8230$ $384487,-0.6608340 \quad 032,-1.0427025372 \backslash \mathrm{C}, 3.3627511633,-1.2189897087,1.2399635578 \backslash \mathrm{H}, 3.451$ $5074863,-2.3051262367,1.3622542861 \backslash \mathrm{H}, 3.0075884152,-0.8036006695,2.1895739234 \backslash \mathrm{H}, 4.34$ $96474965,-0.8147837972,1.00889325 \backslash \backslash$ Version$=I A 64 L-G 03 R e v C .02 \backslash$ State $=2-A \backslash H F=-688.3320$ $429 \backslash \mathrm{S} 2=0.764924 \backslash \mathrm{S} 2-1=0 . \backslash \mathrm{S} 2 \mathrm{~A}=0.750061 \backslash \mathrm{RMSD}=7.129 \mathrm{e}-09 \backslash \mathrm{RMSF}=2.171 \mathrm{e}-06 \backslash \mathrm{Dipole}=-0.982$ $4654,0.3847166,0.726171 \backslash \mathrm{PG}=\mathrm{C} 01[\mathrm{X}(\mathrm{C} 9 \mathrm{H} 1$ 7N2O3)]\\@,

\section{$\mathrm{CH}_{2} \mathrm{CH}\left(\mathrm{CH}_{3}\right)_{2}$}

$1 \backslash 1 \backslash$ GINC-AC49\FOpt $\backslash U B 3 L Y P \backslash 6-31 G(d) \backslash C 4 H 9(2) \backslash G P W 501 \backslash 30-N o v-2005 \backslash 0 \backslash \ \#$ B3L YP/6-31G( D) OPT FREQ $\mid \backslash$ comment $\backslash 0,2 \backslash C, 0.8846012921,-0.9464836033,0.7390001952 \backslash \mathrm{H}, 1.7935589616$,$1.1023215379,0.1639512775 \backslash \mathrm{H}, 0.9138365549,-1.1873093591,1.7980542705 \backslash \mathrm{C},-0.2735263584$,$0.1971879713,0.1578985957 \backslash \mathrm{C},-0.2549754283,1.290443148,0.5900410126 \backslash \mathrm{H},-1.2019167915,-0$ $.6312182804,0.5632781618 \backslash \mathrm{C},-0.3188458004,-0.3193080099,-1.3733847246 \backslash \mathrm{H}, 0.6386834438,1$ $.795622806,0.2045031599 \backslash \mathrm{H},-0.2504465587,1.3866075136,1.6814804266 \backslash \mathrm{H},-1.1371158618,1$. $8181061958,0.205333313 \backslash \mathrm{H},-0.3835754754,-1.3674759863,-1.685848305 \backslash \mathrm{H}, 0.5857346559,0.10$ $95183278,-1.8237452148 \backslash \mathrm{H},-1.1822811591,0.2136889397,-1.7883375627 \backslash \backslash$ Version=IA64L-G03 RevC.02 $\backslash$ State $=2-\mathrm{A} \backslash \mathrm{HF}=-157.7853957 \backslash \mathrm{S} 2=0.753844 \backslash \mathrm{S} 2-1=0 . \backslash \mathrm{S} 2 \mathrm{~A}=0.750009 \backslash \mathrm{RMSD}=9.525 \mathrm{e}-09$ $\backslash \mathrm{RMSF}=1.630 \mathrm{e}-06 \backslash \mathrm{Di}$ pole=-0.0461221,0.0660729,0.0045471 $\backslash \mathrm{PG}=\mathrm{C} 01$ [X(C4H9)]\\@ 


\section{TABLE S5: GAUSSIAN Archive Entries for B3-LYP/6-31G(d) Optimized Equilibrium Structures of Additional Species Involved in Reactions of the Valine Residue}

Reactant $\mathrm{CH}_{3} \mathrm{CONHCO}\left(\mathrm{CH}\left(\mathrm{CH}_{3}\right)_{2}\right) \mathrm{CONHCH}_{3}$

$1 \backslash 1 \backslash$ GINC-AC28\FOpt $\backslash U B 3 L Y P \backslash 6-31 G(d) \backslash C 8 H 15 N 2 O 3(2) \backslash G P W 501 \backslash 16-F e b-2006 \backslash 0 \backslash \backslash \#$ b3lyp/6 $-31 \mathrm{G}(\mathrm{d})$ opt freq $\backslash \backslash \mathrm{comment} \backslash \backslash 0,2 \backslash \mathrm{C},-0.1329510283,1.6237662652,0 \quad .2587249175 \backslash \mathrm{C},-0.096490730$ $3,0.1241753339,-0.3056871526 \backslash \mathrm{N}, 0.9595152732,-0.6932881179,0.2862659159 \backslash \mathrm{O},-0.14429353$ $35,0.1174658427,-1.6309728301 \backslash \mathrm{C},-1.4732141377,-0.5540873755,0.2024060999 \backslash \mathrm{H}, 0.6586985$ $973,-1.2927014728,1.0442634866 \backslash \mathrm{C}, 2.2452547224,-0.7895915583,-0.1822993341 \backslash \mathrm{O}, 2.700696$ $0076,-0.0895646221,-1.0754037547 \backslash \mathrm{C}, 3.0796212344,-1.8707501111,0.4964285593 \backslash \mathrm{H}, 2.7203$ $27503,-2.1335769345,1.496748383 \backslash \mathrm{H}, 4.1141634481,-1.526494668 \quad 7,0.5588703575 \backslash \mathrm{H}, 3.063818$ $5535,-2.7749521704,-0.1232917299 \backslash \mathrm{O},-1.5040492531,-1.1710305042,1.2557769496 \backslash \mathrm{N},-2.5032$ $134299,-0.3281276884,-0.6276773474 \backslash \mathrm{H},-2.2665791725,0.1318257726,-1.4992586682 \backslash \mathrm{C},-3.85$ $70537594,-0.794 \quad 0827766,-0.3733487198 \backslash \mathrm{H},-4.1941952853,-1.4499905933,-1.1829905577 \backslash \mathrm{H},-4$ $.5504710628,0.049597775,-0.2865457383 \backslash \mathrm{H},-3.8546432144,-1.3533358292,0.5628371279 \backslash \mathrm{H}$, $-1.1200207091,2.0016568752,-0.0365866476 \backslash \mathrm{C}, 0.935425831,2$. 486274354,-0.425704091\C,-0. $0118177691,1.6755915228,1.7844414026 \backslash \mathrm{H}, 0.8 \quad 602706804,3.5152360837,-0.0531477354 \backslash \mathrm{H}, 0$. $7993153266,2.4951700536,-1.51 \quad 04001769 \backslash \mathrm{H}, 1.9430313894,2.114281884,-0.225996477 \backslash \mathrm{H},-0.7$ $57970942,1.0545573014,2.288567631 \backslash \mathrm{H},-0.1461052866,2.7098763252,2.1222383129 \backslash \mathrm{H}, 0.980$ $870747,1.3450790339,2.1073618171 \backslash \backslash$ Version=IA64L-G03RevD.01 $\backslash$ State $=2-A \backslash H F=-649.0308$ $829 \backslash \mathrm{S} 2=0.754833 \backslash \mathrm{S} 2-1=0 . \backslash \mathrm{S} 2 \mathrm{~A}=0.750014 \backslash \mathrm{RMSD}=8.490 \mathrm{e}-09 \backslash \mathrm{RMSF}=2.643 \mathrm{e}-05 \backslash$ Thermal $=0 . \backslash \mathrm{Di}$ pole $=-1.5116525,-0.1356981,0.5734172 \backslash \mathrm{PG}=\mathrm{C} 01[\mathrm{X}(\mathrm{C} 8 \mathrm{H} 15 \mathrm{~N} 2 \mathrm{O} 3)] \backslash \backslash @$

Transition Structure Pathway A $\mathrm{CH}_{3} \mathrm{CONHCO}\left(\mathrm{CH}\left(\mathrm{CH}_{3}\right)_{2}\right)--\mathrm{CONHCH}_{3}$

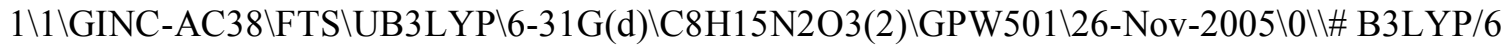
$-31 \mathrm{G}(\mathrm{D}) \mathrm{OPT}=(\mathrm{TS}, \mathrm{NOEIGENTEST,CALCFC,MAXCYC=100)} \mathrm{FREQ} \backslash \backslash$ comment $\backslash \backslash 0,2 \backslash \mathrm{C},-1.302$ $4330917,-0.3238865808,0.2428475438 \backslash \mathrm{C}, 0.2783200323,0.6157917401,-0.435615871 \backslash \mathrm{O},-1.3767$ $269065,-0.587339328,1.4162648311 \backslash \mathrm{N},-2.1398235296,-0.5150325738,-0.7653764694 \backslash \mathrm{H},-1.731$ $390097,-0.2939007949,-1.670760701 \backslash \mathrm{C},-3.3587054335,-1.3105189522,-0.6551662081 \backslash \mathrm{H},-4.116$ $1732693,-0.9056691219,-1.3316311804 \backslash \mathrm{H},-3.7276319457,-1.2538171052,0.3703991872 \backslash \mathrm{H},-3.1$ $693555336,-2.3595417748,-0.9096748914 \backslash \mathrm{C}, 0.1085701026,2.0401303933,0.177443235 \backslash \mathrm{N}, 1.34$ $60947626,-0.1393126668,0.1712273609 \backslash \mathrm{O}, 0.0759295272,0.4949391475,-1.6799789983 \backslash \mathrm{H}, 1.78$ $98915895,0.2708141159,0.9818128237 \backslash \mathrm{C}, 1.5289109212,-1.5004814188,-0.0108934737 \backslash \mathrm{O}, 0.81$ $66170168,-2.17817312,-0.7313766196 \backslash \mathrm{C}, 2.6714626075,-2.0910727968,0.8042078007 \backslash \mathrm{H}, 3.5179$ $519398,-1.4029301177,0.9002959545 \backslash \mathrm{H}, 3.0025635729,-3.0134936239,0.3240396346 \backslash \mathrm{H}, 2.3141$ $511021,-2.339747761,1.8113149472 \backslash \mathrm{H},-0.9119089432,2.3365642293,-0.0932500188 \backslash \mathrm{C}, 1.07618$ $14811,2.9944455913,-0.5497137392 \backslash \mathrm{C}, 0.2619804635,2.1457149133,1.7010413002 \backslash \mathrm{H}, 0.914661$ $9225,4.0259239552,-0.2158474076 \backslash \mathrm{H}, 0.922657124,2.9456097062,-1.6308392368 \backslash \mathrm{H}, 2.1214674$ $55,2.7349708347,-0.3412961453 \backslash \mathrm{H},-0.3459001444,1.4088495986,2.2316643487 \backslash \mathrm{H},-0.04491558$ $64,3.1445674391,2.0315069696 \backslash \mathrm{H}, 1.3077525847,2.026066173,2.017132242 \backslash \backslash$ Version=I A64LG03RevC.02 $\backslash$ State $=2-A \backslash H F=-649.0253768 \backslash \mathrm{S} 2=0.75999 \backslash \mathrm{S} 2-1=0 . \backslash \mathrm{S} 2 \mathrm{~A}=0.750036 \backslash \mathrm{RMSD}=5.352 \mathrm{e}$ $-09 \backslash \mathrm{RMSF}=2.425 \mathrm{e}-06 \backslash$ Dipole=-0.3215638,0.5706395,0.697226 2\PG=C01 [X(C8H15N2O3)]\\@

\section{$\mathrm{CH}_{3} \mathrm{CONHCO}\left(\mathrm{CH}\left(\mathrm{CH}_{3}\right)_{2}\right)$}


$1 \backslash 1 \backslash$ GINC-AC25 $\backslash F O p t \backslash R B 3 L Y P \backslash 6-31 G(d) \backslash C 6 H 11 N 1 O 2 \backslash G P W 501 \backslash 16-F e b-2006 \backslash 0 \backslash \backslash \#$ B 3LYP/6$31 \mathrm{G}(\mathrm{D})$ opt freq $\backslash \backslash$ comment $\backslash \backslash 0,1 \backslash \mathrm{C},-0.0692139651,1.6722956323,0.1484176674 \backslash \mathrm{C}, 0.353037695$ $7,0.3883434735,-0.5827493509 \backslash \mathrm{N}, 1.461621633,-0.25 \quad 59026825,-0.0066221718 \backslash \mathrm{O},-0.22312471$ $26,-0.0230700636,-1.5652134779 \backslash \mathrm{H}, 1 \quad .8572212908,0.1853447113,0.8133441666 \backslash \mathrm{C}, 2.08989786$ 27,-1.441154717,-0.4 305971978\O,1.7382029524,-2.0863164395,-1.3910783826\C,3.2683918 $661,-1.8289272211,0.4544082584 \backslash \mathrm{H}, 4.0339243547,-1.0434012193,0.4591055407 \backslash \mathrm{H}, 3 \quad .704025$ $6351,-2.7503697322,0.066925277 \backslash \mathrm{H}, 2.9472265548,-1.9910283825,1.4906043959 \backslash \mathrm{H},-0.927737$ $691,2.0241111873,-0.4324490413 \backslash \mathrm{C}, 1.0263199116,2.753228811,0.1011482738 \backslash \mathrm{C},-0.53758647$ $24,1.3920735697,1.5881901825 \backslash \mathrm{H}, 0.64 \quad 46840725,3.6930912861,0.5153820408 \backslash \mathrm{H}, 1.354508762$ $1,2.9446436209,-0.9259107059 \backslash \mathrm{H}, 1.9092571439,2.4746668427,0.6907550653 \backslash \mathrm{H},-1.315049483$ $8,0.621253212,1.6123960727 \backslash \mathrm{H},-0.9511100409,2.3041504381,2.0328138289 \backslash \mathrm{H}, 0.285332630$ $3,1.0612276727,2.2348395582 \backslash \backslash$ Version $=I A 64 L-G 03 R e v D .01 \backslash$ State $=1-A \backslash H F=-440.4866501 \backslash \mathrm{R}$ $\mathrm{MSD}=5.089 \mathrm{e}-09 \backslash \mathrm{RMSF}=7.827 \mathrm{e}-07 \backslash \mathrm{Thermal}=0 . \mid \mathrm{Dipole}=0.8356205,1.1277904,1.9114828 \backslash \mathrm{PG}=$ C01 [X(C6H11N1O2)]\\@

Transition Structure Pathway B $\mathrm{CH}_{3} \mathrm{CONH}-\mathrm{CO}\left(\mathrm{CH}\left(\mathrm{CH}_{3}\right)_{2}\right) \mathrm{CONHCH}_{3}$

$1 \backslash 1 \backslash$ GINC-AC51\FTS $\backslash U B 3 L Y P \backslash 6-31 G(d) \backslash C 8 H 15 N 2 O 3(2) \backslash G P W 501 \backslash 30-N o v-2005 \backslash 0 \backslash$ \# B3LYP/6 $-31 \mathrm{G}(\mathrm{D}) \mathrm{OPT}=(\mathrm{CALCFC}, \mathrm{MAXCYC}=100, \mathrm{TS}, \mathrm{NOEIGENTEST}) \mathrm{FREQ} \backslash \backslash$ comment $\backslash \backslash 0,2 \backslash \mathrm{N}, 0.7503$ $766291,-0.7840059237,0.9022773925 \backslash \mathrm{C},-0.2821440965,0.5731453392,-0.5225101622 \backslash \mathrm{H}, 0.6564$ $5035,-1.094431383,1.8662213867 \backslash \mathrm{C}, 1.7346847867,-1.3346060154,0.2549181473 \backslash \mathrm{O}, 2.0689027$ $704,-1.0964853935,-0.9540650187 \backslash \mathrm{H}, 2.3467440244,-2.5927010744,1.9355663086 \backslash \mathrm{C}, 2.6615399$ $728,-2.3783798872,0.9119894277 \backslash \mathrm{H}, 3.6857948792,-1.9957021994,0.9156188976 \backslash \mathrm{H}, 2.6442367$ $44,-3.2973060122,0.3201623597 \backslash \mathrm{C},-1.6884974078,0.2272063688,0.0823578664 \backslash \mathrm{O},-2.23059744$ $73,0.9297505207,0.9260147898 \backslash \mathrm{N},-2.2165153931,-0.8797809788,-0.4794652972 \backslash \mathrm{H},-1.6366421$ $486,-1.33053223,-1.17714476 \backslash \mathrm{C},-3.5081945937,-1.4236121786,-0.1124060527 \backslash \mathrm{H},-4.142452157$ $8,-1.5472596407,-0.9973825257 \backslash \mathrm{H},-3.9848649143,-0.7224822601,0.5746308705 \backslash \mathrm{H},-3.4058218$ $122,-2.3959195874,0.3853466627 \backslash \mathrm{C}, 0.3167343702,1.9306997326,-0.1547304499 \backslash \mathrm{O}, 0.0282888$ $601,-0.0413082973,-1.5510529673 \backslash \mathrm{H},-0.4903665001,2.61980379,-0.458957074 \backslash \mathrm{C}, 1.559492505$ $4,2.2325177183,-0.9992107932 \backslash \mathrm{C}, 0.5626346653,2.1735347808,1.3412463697 \backslash \mathrm{H}, 1.9009180574$

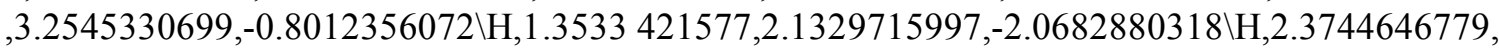
$1.5431284896,-0.7579289829 \backslash \mathrm{H},-0.3180469536,1.9205213804,1.9332828836 \backslash \mathrm{H}, 0.79620819,3.2$ $325619177,1.5024332743 \backslash \mathrm{H}, 1.4127520722,1.5806326672,1.690889122 \backslash \backslash$ Version=IA64 L-G03R evC.02 $\backslash$ State $=2-\mathrm{A} \backslash \mathrm{HF}=-648.9906119 \backslash \mathrm{S} 2=0.756813 \backslash \mathrm{S} 2-1=0 . \backslash \mathrm{S} 2 \mathrm{~A}=0.750036 \backslash \mathrm{RMSD}=9.379 \mathrm{e}-09 \backslash$ $\mathrm{RMSF}=6.658 \mathrm{e}-06 \backslash \mathrm{Dipole}=0.1216167,-1.1292199,0.6199143 \backslash \mathrm{PG}=\mathrm{C} 01[\mathrm{X}(\mathrm{C} 8 \mathrm{H} 15 \mathrm{~N} 2 \mathrm{O} 3)] \backslash \backslash @$

\section{$\mathrm{CO}\left(\mathrm{CH}\left(\mathrm{CH}_{3}\right)_{2}\right) \mathrm{CONHCH}_{3}$}

$1 \backslash 1 \backslash$ GINC-AC30\FOpt $\backslash R B 3 L Y P \backslash 6-31 G(d) \backslash C 6 H 11 N 1 O 2 \backslash G P W 501 \backslash 16-F e b-2006 \backslash 0 \backslash \backslash \#$ B 3LYP/6$31 \mathrm{G}(\mathrm{D})$ opt freq $\backslash \backslash \mathrm{comment} \backslash \backslash 0,1 \backslash \mathrm{C},-0.1099900449,1.9479685008,0.41 \quad 74433392 \backslash \mathrm{C},-0.752399337$ $3,0.8483855535,-0.4040820991 \backslash \mathrm{O},-0.2379981614,0$. 3374072408,-1.3845938751 \C,-2.1432650 $64,0.351755428,0.0702553259 \backslash \mathrm{O},-2.7215732406,0.8595151042,1.0268812493 \backslash \mathrm{N},-2.604793958$ $6,-0.6684024246,-0 . \quad 6877362885 \backslash \mathrm{H},-2.0011439701,-0.9553371485,-1.4491137394 \backslash \mathrm{C},-3.884514$ $9195,-1.3106610043,-0.4656352754 \backslash \mathrm{H},-3.7575338774,-2.3785230165,-0.2525741643 \backslash \mathrm{H},-4.53$ $77505733,-1.2015202771,-1.3393196328 \backslash \mathrm{H},-4.3524571733,-0.8271 \quad 376683,0.3935945878 \backslash \mathrm{H},-0$. $9115786727,2.6427725605,0.6961703287 \backslash \mathrm{C}, 0.9820 \quad 402285,2.6712993024,-0.3746462326 \backslash \mathrm{C}, 0.4$ $339227275,1.3345393715,1.7302973034 \backslash \mathrm{H}, 1.4269415403,3.4655451238,0.2343455687 \backslash \mathrm{H}, 0.58$ $11149296,3.1241778347,-1.2875740281 \backslash \mathrm{H}, 1.7746978272,1.9771495441,-0.6711419329 \backslash \mathrm{H},-0.3$ 
$657197713,0.8588756757,2.3041078224 \backslash \mathrm{H}, 0.8751961748,2.1244052231,2.3475383531 \backslash \mathrm{H}, 1.2$ $136853366,0.5927450764,1.5211933897 \backslash \backslash$ Version=IA64L-G03RevD.01 $\backslash$ State $=1-A \backslash H F=-440.47$ $87584 \backslash$ RMSD $=9.077 \mathrm{e}-09 \backslash \mathrm{RMSF}=4.553 \mathrm{e}-06 \backslash$ Thermal $=0 . \backslash$ Dip ole $=-0.0804559,-0.4217026,-0.270$ 3188\PG=C01 [X(C6H11N1O2)]\@,

Transition Structure Pathway $\mathrm{C} \mathrm{CH}_{3} \mathrm{CONHCO}--\left(\mathrm{CH}\left(\mathrm{CH}_{3}\right)_{2}\right) \mathrm{CONHCH}_{3}$

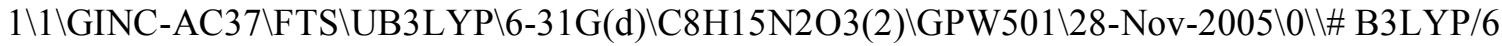
$-31 \mathrm{G}(\mathrm{D}) \mathrm{OPT}=(\mathrm{CALCFC}, \mathrm{TS}, \mathrm{NOEIGENTEST,MAXCYC=100)} \mathrm{FREQ} \backslash \backslash$ commnet $\backslash 0,2 \backslash \mathrm{C},-0.146$ $4392232,1.7284995673,0.3960095982 \backslash \mathrm{C},-0.1384442989,-0.0556358377,-0.4497675047 \backslash \mathrm{H},-1.19$ $04247659,1.9648858625,0.1828636098 \backslash \mathrm{C}, 0.1420397705,1.5510221389,1.8649783235 \backslash \mathrm{H},-0.503$ $0504001,0.8014588956,2.3306185283 \backslash \mathrm{H}, 1.1885480909,1.2791313233,2.0359765514 \backslash \mathrm{H},-0.0366$ $089416,2.5110613809,2.3732104003 \backslash \mathrm{H}, 0.859662971,3.5785855663,0.0696214285 \backslash \mathrm{C}, 0.8279742$ $152,2.5756589083,-0.3832432839 \backslash \mathrm{H}, 0.5285872194,2.6713362793,-1.4283041868 \backslash \mathrm{H}, 1.8371763$ $428,2.1561413652,-0.3599684113 \backslash \mathrm{N}, 0.9368540354,-0.7795600183,0.1513649923 \backslash \mathrm{H}, 0.6397580$ $042,-1.3434510105,0.940627532 \backslash \mathrm{C}, 2.2677060845,-0.7944660047,-0.2241838514 \backslash \mathrm{O}, 2.75053271$ $26,-0.0451140072,-1.0554414037 \backslash \mathrm{H}, 2.794122789,-1.9916707918,1.536365237 \backslash \mathrm{C}, 3.0874275897$ ,-1.863873439, $0.4884308728 \backslash \mathrm{H}, 4.1423918596,-1.5902265242,0.4342230173 \backslash \mathrm{H}, 2.9526916587,-$ $2.8275665041,-0.0173981144 \backslash \mathrm{C},-1.4727731219,-0.5870982746,0.167516937 \backslash \mathrm{O},-1.4981520657$, $-1.1779176644,1.2461175006 \backslash \mathrm{N},-2.532295789,-0.3265777793,-0.6215065143 \backslash \mathrm{H},-2.3006101787$ $, 0.0791956795,-1.5218105326 \backslash \mathrm{C},-3.8834914841,-0.7565509043,-0.3150858966 \backslash \mathrm{H},-4.57858110$ $66,0.0891428029,-0.3567770035 \backslash \mathrm{H},-3.8825892318,-1.1726754911,0.6936518932 \backslash \mathrm{H},-4.2227914$ $252,-1.5270879007,-1.0174036002 \backslash \mathrm{O},-0.1479041221,0.2579525122,-1.6747307049 \backslash \backslash$ Ve rsion=I A64L-G03RevC.02 $\backslash$ State $=2-A \backslash H F=-649.0247794 \backslash \mathrm{S} 2=0.762256 \backslash \mathrm{S} 2-1=0 . \backslash \mathrm{S} 2 \mathrm{~A}=0.750046 \backslash \mathrm{RMSD}$ $=4.797 \mathrm{e}-09 \backslash \mathrm{RMSF}=4.430 \mathrm{e}-06 \backslash \mathrm{Dipole}=-1.0886028,0.0607087,0.7933915 \backslash \mathrm{PG}=\mathrm{C} 01 \quad[\mathrm{X}(\mathrm{C} 8 \mathrm{H} 15 \mathrm{~N} 2$ O3)]1\@

$\mathrm{CH}\left(\mathrm{CH}_{3}\right)_{2}$

$1 \backslash 1 \backslash$ GINC-AC47\FOpt $\backslash$ UB3LYP $\backslash 6-31 G(d) \backslash C 3 H 7(2) \backslash G P W 501 \backslash 16-F e b-2006 \backslash 0 \backslash \backslash \#$ B3L YP/6-31G( D) opt freq $\backslash$ comment $\backslash \backslash 0,2 \backslash \mathrm{C},-0.1577747185,1.8976239731,0.3468 \quad 156712 \backslash \mathrm{H},-1.145580012,1.81$ $58375048,-0.1013806746 \backslash \mathrm{C}, 0.9334283418,2.5333812999,-0.4490231684 \backslash \mathrm{C},-0.0265372247,1.6$ $946451576,1.8198604193 \backslash \mathrm{H}, 0.992 \quad 6405348,3.6249832325,-0.2807754821 \backslash \mathrm{H}, 0.7932167498,2.3$ $872408347,-1.5261558015 \backslash \mathrm{H}, 1.9192496019,2.1301067917,-0.1767902975 \backslash \mathrm{H},-0.7878506807,1$. $008438488,2.2080911352 \backslash \mathrm{H},-0.1327211238,2.6402639781,2.3837162063 \backslash \mathrm{H}, 0.9612685314,1$. $2918187397,2.0855119922 \backslash \backslash$ Version=IA64L-G03RevD.01 $\backslash$ State $=2-A \backslash H \quad F=-118.4781528 \backslash \mathrm{S} 2=0$. $754003 \backslash \mathrm{S} 2-1=0 . \backslash \mathrm{S} 2 \mathrm{~A}=0.750011 \backslash \mathrm{RMSD}=5.496 \mathrm{e}-09 \backslash \mathrm{RMSF}=9.021 \mathrm{e}-06 \backslash \mathrm{Thermal}=0 . \backslash \mathrm{Dipole}=0.00$ 73893,0.073684,0.0303382 $\backslash \mathrm{PG}=\mathrm{C} 01[\mathrm{X}(\mathrm{C} 3 \mathrm{H} 7)] 1 \backslash @$ 


\title{
TABLE S6: GAUSSIAN Archive Entries for B3-LYP/6-31G(d) Optimized Equilibrium Structures of Additional Species Involved in Reactions of the Phenylalanine Residue
}

\author{
Reactant $\mathrm{CH}_{3} \mathrm{CONHCO}\left(\mathrm{CH}_{2}\right.$-phe $) \mathrm{CONHCH}_{3}$
}

$1 \backslash 1 \backslash$ GINC-AC44\FOpt $\backslash U B 3 L Y P \backslash 6-31 G(d) \backslash C 12 H 15 N 2 O 3(2) \backslash G P W 501 \backslash 16-F e b-2006 \backslash 0 \backslash \backslash \#$ B3LYP $/ 6-31 \mathrm{G}(\mathrm{d})$ opt freq $\backslash \backslash$ comment $\backslash \backslash 0,2 \backslash \mathrm{C},-0.9990333024,0.1479235521,-0.2522960416 \backslash \mathrm{C},-0.1952840$ $465,-0.8209506338,0.7357675855 \backslash \mathrm{O},-0.8030031549,-0.1602364792,-1.5216082374 \backslash \mathrm{N},-2.39646$ $64407,0.2106112795,0.1632036442 \backslash \mathrm{H},-2.6611373492,1.0190874853,0.7112393641 \backslash \mathrm{C},-3.25957$ $49895,-0.8441652762,0.0176946216 \backslash \mathrm{C},-0.4092852675,1.6169793339,0.0174972692 \backslash \mathrm{O},-0.85014$ $87911,2.2832361906,0.942042464 \backslash \mathrm{O},-2.9305566644,-1.9136266203,-0.4797707929 \backslash \mathrm{C},-4.6815$ $504014,-0.5815850163,0.4952175197 \backslash \mathrm{H},-5.0449977125,-1.467814803,1.0212274862 \backslash \mathrm{H},-5.3238$ $299473,-0.4284962721,-0.3792395337 \backslash \mathrm{H},-4.7705148852,0.2929713336,1.1472742422 \backslash \mathrm{C}, 1.2823$ $394105,-0.9219054991,0.4824209294 \backslash \mathrm{H},-0.695454059,-1.7838384614,0.5998338414 \backslash \mathrm{H},-0.3979$ $89044,-0.4585431471,1.7480820275 \backslash \mathrm{C}, 4.0523676596,-1.111695308,0.0211382857 \backslash \mathrm{C}, 1.778479$ $7173,-1.6563287826,-0.6066695073 \backslash \mathrm{C}, 2.1978842812,-0.2849969576,1.3336452931 \backslash \mathrm{C}, 3.571971$ $3435,-0.3789317638,1.1079114126 \backslash \mathrm{C}, 3.1509007364,-1.7500810807,-0.8350103637 \backslash \mathrm{H}, 1.078924$ $8783,-2.1475063752,-1.27680038 \backslash \mathrm{H}, 1.8279270389,0.2834143468,2.1843012302 \backslash \mathrm{H}, 4.26580335$ $6,0.1146774087,1.7835111181 \backslash \mathrm{H}, 3.5181724359,-2.325641731,-1.6806641671 \backslash \mathrm{H}, 5.1218604597$, $-1.1887286922,-0.1560282793 \backslash \mathrm{N}, 0.5653549433,1.9597802711,-0.8341360206 \backslash \mathrm{H}, 0.8127310242$ $, 1.2569741783,-1.5208714615 \backslash \mathrm{C}, 1.361710343,3.1676953102,-0.6900501484 \backslash \mathrm{H}, 0.8098968942,3$ $.873832102,-0.0680941023 \backslash \mathrm{H}, 1.5373524924,3.6119761068,-1.6738721866 \backslash \mathrm{H}, 2.3263990415,2.9$ $503340007,-0.2166271125 \backslash \backslash$ Version $=I$ A64L-G03RevD.01 $\backslash$ State $=2-A \backslash H F=-801.4582003 \backslash \mathrm{S} 2=0.7$ $55156 \backslash \mathrm{S} 2-1=0 . \mathrm{S} 2 \mathrm{~A}=0.750016 \backslash \mathrm{RMSD}=5.019 \mathrm{e}-09 \backslash \mathrm{RMSF}=3.014 \mathrm{e}-06 \backslash$ Thermal $=0 . \backslash \mathrm{Dipole}=0.9629$ 66,1.169021 3,0.2995016 $\mathrm{PG}=\mathrm{C} 01$ [X(C12H15N2O3)]\@

Transition Structure Pathway A $\mathrm{CH}_{3} \mathrm{CONHCO}\left(\mathrm{CH}_{2}\right.$-phe)-- $\mathrm{CONHCH}_{3}$

$1 \backslash 1 \backslash$ GINC-AC38\FTS $\backslash U B 3 L Y P \backslash 6-31 G(d) \backslash C 12 H 15 N 2 O 3(2) \backslash G P W 501 \backslash 05-D e c-2005 \backslash 0 \backslash \backslash$ \# B3LYP /6-31G(D) FREQ INT(GRID=ULTRAFINE) OPT=(TS,CALCFC,NOEIGENTEST) \〈comment $\backslash \backslash$ $0,2 \backslash \mathrm{O},-1.2003169456,-0.1115745426,-1.8385761428 \backslash \mathrm{C},-0.9095690 \quad 486,0.6439830102,-0.94581$ $01066 \backslash \mathrm{C}, 0.8133753737,0.4207809606,0.0117977342 \backslash \mathrm{N},-1.493550527,1.7211522182,-0.447600$ $1691 \backslash \mathrm{H},-0.9368431641,2.22879527 \quad 27,0.237223808 \backslash \mathrm{C},-2.6800490665,2.3374159775,-1.035157$ $5828 \backslash \mathrm{H},-3.1791761 \quad 723,1.6041140438,-1.6704053826 \backslash \mathrm{H},-2.4097577822,3.2131902203,-1.6356$ $383801 \backslash \mathrm{H},-3.3622402094,2.6464081009,-0.2381028561 \backslash \mathrm{C}, 1.085182795,-1.091477923,-0.184$ $8929342 \backslash \mathrm{O}, 0.5222637597,0.8901294812,1.1357715229 \backslash \mathrm{N}, 1.6630220 \quad 86,1.1968799891,-0.8845$ $380982 \backslash \mathrm{H}, 2.2011453697,0.6702475243,-1.5601431024 \backslash \mathrm{C}, 1.742314083,2.5743478893,-0.94723$ $48634 \backslash \mathrm{O}, 0.9961709307,3.3263993389,-0.3374287994 \backslash \mathrm{C}, 2.8664706282,3.0963042275,-1.83247$ $05345 \backslash \mathrm{H}, 2.61118812,4.1036641223,-2.16594392 \backslash \mathrm{H}, 3.054510091,2.4596580902,-2.7040554693$ $\backslash \mathrm{H}, 3.793095038,3.1519286569,-1.2487231973 \backslash \mathrm{C}, 0.2055991685,-1.9974738858,0.6436434145$ $\backslash \mathrm{H}, 2.139042445,-1.234735926,0.0960642291 \backslash \mathrm{H}, 0.9915497347,-1.337 \quad 7479877,-1.2474952157 \backslash$ C,-1.4040412596,-3.7153683473,2.181207145\C,0.32 84553087,-2.0317233074,2.0401209334 $\mathrm{C},-0.7377339721,-2.8314164264,0.03 \quad 09843742 \backslash \mathrm{C},-1.5364750508,-3.6863471737,0.792820285$ $9 \backslash \mathrm{C},-0.4699686602,-2.8829852202,2.8025459322 \backslash \mathrm{H}, 1.0448610404,-1.3781293065,2.52807700$ $41 \backslash \mathrm{H},-\quad 0.8521658582,-2.8029846852,-1.0490756072 \backslash \mathrm{H},-2.261456358,-4.3286208308,0.299391$ $3462 \backslash \mathrm{H},-0.3625466448,-2.8966581178,3.8841241144 \backslash \mathrm{H},-2.023810315 \quad 5,-4.3812275369,2.7762$ $15068 \backslash \backslash$ Version=IA64L-G03RevC.02 $\backslash$ State $=2-A \backslash H F=-80 \quad 1.4510775 \backslash \mathrm{S} 2=0.759809 \backslash \mathrm{S} 2-1=0 . \mid \mathrm{S} 2 \mathrm{~A}$ 
$=0.750035 \backslash \mathrm{RMSD}=5.726 \mathrm{e}-09 \backslash \mathrm{RMSF}=2.230 \mathrm{e}-06 \backslash \mathrm{Dipole}=0.3014729,0.1146989,-1.0398751 \backslash \mathrm{PG}$ $=\mathrm{C} 01[\mathrm{X}(\mathrm{C} 12 \mathrm{H} 15 \mathrm{~N} 2 \mathrm{O} 3)] \backslash \backslash @$

\section{$\mathrm{CH}_{3} \mathrm{CONHCO}\left(\mathrm{CH}_{2}\right.$-phe $)$}

$1 \backslash 1 \backslash$ GINC-AC44\FOpt $\backslash R B 3 L Y P \backslash 6-31 G(d) \backslash C 10 H 11 N 1 O 2 \backslash G P W 501 \backslash 16-F e b-2006 \backslash 0 \backslash \backslash \#$ b3lyp/6-31 $\mathrm{G}(\mathrm{d})$ opt freq $\backslash \backslash$ comment $\backslash \backslash 0,1 \backslash \mathrm{C}, 0.2324363894,-0.3783717005,0.3256719872 \backslash \mathrm{O}, 0.4796345595,1$. $4412806408,-1.2660830747 \backslash \mathrm{C}, 0.7481696733,1.0018975835,-0.1510632882 \backslash \mathrm{N}, 1.5066015881,1$. $6131515043,0.7848402357 \backslash \mathrm{H}, 1.6398596674,1.0966588272,1.6462237267 \backslash \mathrm{C}, 2.1238589532,2.90$ $98205806,0.5904014524 \backslash \mathrm{H}, 1.840231595,3.2666203546,-0.4011173259 \backslash \mathrm{H}, 3.2164109481,2.8389$ $418277,0.6476546049 \backslash \mathrm{H}, 1.7771162246,3.6263082606,1.3439105155 \backslash \mathrm{C},-0.7238753564,-1.0952$ $176977,-0.6091352335 \backslash 0,0.5788341426,-0.8171259822,1.4072736574 \backslash \mathrm{C},-2.1518249833,-0.636$ $3694798,-0.3391552742 \backslash \mathrm{H},-0.6265869046,-2.1679058013,-0.419779488 \backslash \mathrm{H},-0.4456603934,-0.87$ $52318291,-1.6425681304 \backslash \mathrm{C},-4.7739886364,0.2109408956,0.1954902911 \backslash \mathrm{C},-2.9271636618,-1.2$ $896146885,0.6275801757 \backslash \mathrm{C},-2.6970683849,0.4570983778,-1.0243922812 \backslash \mathrm{C},-4.0025085349,0$. $8740960801,-0.7606279426 \backslash \mathrm{C},-4.2317772172,-0.8714937842,0.8910790038 \backslash \mathrm{H},-2.5065014779,-$ $2.131635719,1.1719151758 \backslash \mathrm{H},-2.0889802705,0.9825969496,-1.7547800782 \backslash \mathrm{H},-4.4164465748,1$ $.7196118942,-1.3038990779 \backslash \mathrm{H},-4.8246248352,-1.3929608422,1.6380176014 \backslash \mathrm{H},-5.7911665098$, $0.5357137481,0.3979427673 \backslash \backslash$ Version=IA64L-G03RevD.01 $\backslash$ State $=1-A \backslash H F=-592.900205 \backslash$ RMS $\mathrm{D}=5.004 \mathrm{e}-09 \backslash \mathrm{RMSF}=2.956 \mathrm{e}-06 \backslash$ Thermal $=0 . \backslash$ Dipole $=0.2183343,0.3170879,0.4583123 \backslash \mathrm{PG}=\mathrm{C} 01[$ $\mathrm{X}(\mathrm{C} 10 \mathrm{H} 11 \mathrm{~N} 1 \mathrm{O} 2)] \backslash \backslash$ @

Transition Structure Pathway B $\mathrm{CH}_{3} \mathrm{CONH}-\mathrm{CO}\left(\mathrm{CH}_{2}\right.$-phe) $\mathrm{CONHCH}_{3}$

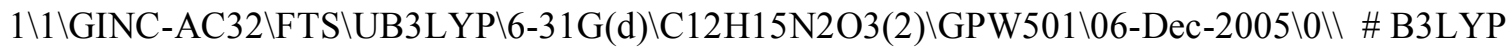
/6-31G(D) OPT=(TS,NOEIGENTEST,CALCFC,MAXCYC=100) IOP(6/7=3) FRE Q INT(GRI $\mathrm{D}=\mathrm{ULTRAFINE}) \backslash \backslash$ comment $\backslash \backslash 0,2 \backslash \mathrm{N}, 2.0650753133,-1.0720750734,-0.6483087534 \backslash \mathrm{C}, 0.2613986$ $562,-0.4364600089,0.3135299034 \backslash \mathrm{H}, 2.0856040232,-0.8 \quad 560627756,-1.6493571194 \backslash \mathrm{C}, 3.300833$ $8958,-0.9465622078,-0.0531840657 \backslash \mathrm{O}, 3.4308254154,-0.6236700131,1.1257539117 \backslash \mathrm{H}, 4.33645$ $16856,-2.1773735425,-1 \quad .5321145191 \backslash \mathrm{C}, 4.5073267485,-1.2784434182,-0.9305191147 \backslash \mathrm{H}, 4.709$ $994783,-0.4475866149,-1.6181739971 \backslash \mathrm{H}, 5.3785691223,-1.4262988747,-0.2890667446 \backslash \mathrm{O}, 0.4$ $682212592,1.4469917459,-1.2613008976 \backslash \mathrm{C}, 0.8039118296,1.0046156926,-0.1702369668 \backslash \mathrm{N}, 1.4$ $915010877,1.6311961061,0.7912340352 \backslash \mathrm{H}, 1.7667039665,1.0468420366,1.5751749205 \backslash \mathrm{C}, 2.10$ $78108664,2.9338773839,0.6074546997 \backslash \mathrm{H}, 1 \quad .6738024494,3.3995167335,-0.2784615988 \backslash \mathrm{H}, 3.19$ $23349193,2.8411924926,0.4 \quad 727967339 \backslash \mathrm{H}, 1.9128773654,3.567220053,1.4783468466 \backslash \mathrm{C},-0.722$ $9605167,-1.1 \quad 046627213,-0.6453546015 \backslash 0,0.3052122912,-0.6703726559,1.5058948297 \backslash \mathrm{C},-2 \quad$. $1433462063,-0.6592951965,-0.3569241318 \backslash \mathrm{H},-0.6159160928,-2.1828347391,-0.4972235239 \backslash \mathrm{H}$ $,-0.4439242965,-0.8566352054,-1.6707603175 \backslash \mathrm{C},-4.7677870539,0.1735444255,0.1954927915 \backslash$ C,-2.875277703,-1.2523023876,0.681208880 $8 \backslash \mathrm{C},-2.7377456443,0.3641420737,-1.1070431992 \backslash$ C,-4.0431616552,0.7755433 857,-0.834426675\C,-4.178928263,-0.8404694405,0.9547206761 \ $\mathrm{H},-2.4157043129,-2.0355209765,1.2783305855 \backslash \mathrm{H},-2.1688021667,0.8416703918,-1.89973230$ $2 \backslash \mathrm{H},-4.4934878099,1.5670369977,-1.4278086972 \backslash \mathrm{H},-4.7358257164,-1.3119888103,1.7601921$ $766 \backslash \mathrm{H},-5.7852341773,0.492217506,0.4062886522 \backslash \backslash$ Version $=$ IA64L-G03RevC.02 $\backslash$ State $=2-A \backslash H$ $\mathrm{F}=-801.4194693 \backslash \mathrm{S} 2=0.770931 \backslash \mathrm{S} 2-1=0 . \backslash \mathrm{S} 2 \mathrm{~A}=0.7 \quad 50186 \backslash \mathrm{RMSD}=6.460 \mathrm{e}-09 \backslash \mathrm{RMSF}=9.637 \mathrm{e}-07 \backslash \mathrm{Di}$ pole $=0.4026599,0.3858345,-0.8394$ 965 $\backslash \mathrm{PG}=\mathrm{C} 01[\mathrm{X}(\mathrm{C} 12 \mathrm{H} 15 \mathrm{~N} 2 \mathrm{O} 3)] \backslash \backslash$ @

\section{$\mathrm{CO}\left(\mathrm{CH}_{2}\right.$-phe $) \mathrm{CONHCH}_{3}$}

$1 \backslash 1 \backslash$ GINC-AC44\FOpt $\backslash R B 3 L Y P \backslash 6-31 G(d) \backslash C 10 H 11 N 1 O 2 \backslash G P W 501 \backslash 16-F e b-2006 \backslash 0 \backslash \backslash \#$ b3lyp/6-31 $\mathrm{G}(\mathrm{d})$ opt freq $\backslash \backslash$ comment $\backslash \backslash 0,1 \backslash \mathrm{C}, 0.2324363894,-0.3783717005,0.3256719872 \backslash \mathrm{O}, 0.4796345595,1$. $4412806408,-1.2660830747 \backslash \mathrm{C}, 0.7481696733,1.0018975835,-0.1510632882 \backslash \mathrm{N}, 1.5066015881,1$. 
$6131515043,0.7848402357 \backslash \mathrm{H}, 1.6398596674,1.0966588272,1.6462237267 \backslash \mathrm{C}, 2.1238589532,2.90$ $98205806,0.5904014524 \backslash \mathrm{H}, 1.840231595,3.2666203546,-0.4011173259 \backslash \mathrm{H}, 3.2164109481,2.8389$ $418277,0.6476546049 \backslash \mathrm{H}, 1.7771162246,3.6263082606,1.3439105155 \backslash \mathrm{C},-0.7238753564,-1.0952$ $176977,-0.6091352335 \backslash \mathrm{O}, 0.5788341426,-0.8171259822,1.4072736574 \backslash \mathrm{C},-2.1518249833,-0.636$ $3694798,-0.3391552742 \backslash \mathrm{H},-0.6265869046,-2.1679058013,-0.419779488 \backslash \mathrm{H},-0.4456603934,-0.87$ $52318291,-1.6425681304 \backslash \mathrm{C},-4.7739886364,0.2109408956,0.1954902911 \backslash \mathrm{C},-2.9271636618,-1.2$ $896146885,0.6275801757 \backslash \mathrm{C},-2.6970683849,0.4570983778,-1.0243922812 \backslash \mathrm{C},-4.0025085349,0$. $8740960801,-0.7606279426 \backslash \mathrm{C},-4.2317772172,-0.8714937842,0.8910790038 \backslash \mathrm{H},-2.5065014779,-$ $2.131635719,1.1719151758 \backslash \mathrm{H},-2.0889802705,0.9825969496,-1.7547800782 \backslash \mathrm{H},-4.4164465748,1$ $.7196118942,-1.3038990779 \backslash \mathrm{H},-4.8246248352,-1.3929608422,1.6380176014 \backslash \mathrm{H},-5.7911665098$, $0.5357137481,0.3979427673 \backslash \backslash$ Version=IA64L-G03RevD.01 $\backslash$ State $=1-A \backslash H F=-592.900205 \backslash$ RMS $\mathrm{D}=5.004 \mathrm{e}-09 \backslash \mathrm{RMSF}=2.956 \mathrm{e}-06 \backslash$ Thermal $=0 . \backslash$ Dipole $=0.2183343,0.3170879,0.4583123 \backslash \mathrm{PG}=\mathrm{C} 01[$ X(C10H11N1O2)]\\@

Transition Structure Pathway C $\mathrm{CH}_{3} \mathrm{CONHCO}--\left(\mathrm{CH}_{2}\right.$-phe) $\mathrm{CONHCH}_{3}$

$1 \backslash 1 \backslash$ GINC-AC32\FTS $\backslash U B 3 L Y P \backslash 6-31 G(d) \backslash C 12 H 15 N 2 O 3(2) \backslash G P W 501 \backslash 04-D e c-2005 \backslash 0 \backslash \backslash$ \# B3LYP /6-31G(D) OPT=(TS,NOEIGENTEST,CALCFC,MAXCYC=100) FREQ INT(GRID= ULTRAFI $\mathrm{NE}) \backslash \backslash$ comment $\backslash \backslash 0,2 \backslash \mathrm{C},-0.1403296859,-0.8828628155,0.7857226934 \backslash \mathrm{C},-1.0740702227,0.261483$ $259,-0.422658099 \backslash \mathrm{H},-0.4188127904,-0.4139229076,1.7283457514 \backslash \mathrm{H},-0.7271056674,-1.764708$ $718,0.5392908196 \backslash \mathrm{C}, 1.2926139428,-0.9588951104,0.5194816234 \backslash \mathrm{C}, 2.2096043593,-0.2148713$ $837,1.2899297354 \backslash \mathrm{H}, 1.8365527278,0.4212171011,2.0890522684 \backslash \mathrm{C}, 1.7884788195,-1.77125678$ $28,-0.5210639779 \backslash \mathrm{H}, 1.0849793537,-2.3256490086,-1.135230025 \backslash \mathrm{C}, 3.155139536,-1.84810541$ $12,-0.7683205369 \backslash \mathrm{H}, 3.5244965921,-2.4805482221,-1.5709733834 \backslash \mathrm{C}, 4.0541416364,-1.1142711$ $052,0.0140226582 \backslash \mathrm{H}, 5.1215892463,-1.1789877893,-0.1791351529 \backslash \mathrm{C}, 3.5775129821,-0.2972454$ $26,1.0436814734 \backslash \mathrm{H}, 4.2733744437,0.2713271646,1.6547466581 \backslash \mathrm{N},-2.4111001827,0.239775351$ $1,0.0946496381 \backslash \mathrm{H},-2.619850317,1.0143100038,0.7155440032 \backslash \mathrm{C},-3.280691345,-0.8318645325$, $0.0307524386 \backslash \mathrm{O},-2.9788675954,-1.9137932401,-0.4472655203 \backslash \mathrm{H},-5.2852510068,-0.09062838$ $2,-0.2046679068 \backslash \mathrm{C},-4.6686236009,-0.5389230259,0.5835079037 \backslash \mathrm{H},-5.1314393135,-1.4793269$ $633,0.8880392837 \backslash \mathrm{H},-4.6510940671,0.1548453934,1.4310341929 \backslash \mathrm{O},-0.8237000289,2.2524824$ $886,0.961705535 \backslash \mathrm{C},-0.4245803319,1.6239056491,-0.0166538456 \backslash \mathrm{N}, 0.5621814806,1.98269099$ $96,-0.8537471819 \backslash \mathrm{H}, 0.7394197246,1.3330189088,-1.6115418266 \backslash \mathrm{C}, 1.3851074038,3.16206502$ 2 2,-0.6671293125\H,0.9597531168,3.7423771539,0.1533141549\H,1.392042422,3.774432148, $-1.5750526183 \backslash \mathrm{H}, 2.4161129748,2.8862789506,-0.4158839174 \backslash \mathrm{O},-0.7997020614,-0.208220412$ $2,-1.5670445173 \backslash \backslash$ Version $=I A 64 L-G 03 R e v C .02 \backslash \mathrm{S}$ tate $=2-A \backslash \mathrm{HF}=-801.452996 \backslash \mathrm{S} 2=0.766254 \backslash \mathrm{S} 2-1$ $=0 . \backslash \mathrm{S} 2 \mathrm{~A}=0.750182 \backslash \mathrm{RMSD}=4.951 \mathrm{e}-09 \backslash \mathrm{RMSF}=1.118 \mathrm{e}-06 \backslash \mathrm{Dipole}=1.0921158,0.8286786,0.43635$ $31 \backslash \mathrm{PG}=\mathrm{C} 01[\mathrm{X}(\mathrm{C} 12 \mathrm{H} 15 \mathrm{~N} 2 \mathrm{O} 3)] \backslash \backslash @$

$\mathrm{CH}_{2}$-phe

$1 \backslash 1 \backslash$ GINC-AC44\FOpt $\backslash U B 3 L Y P \backslash 6-31 G(d) \backslash C 7 H 7(2) \backslash G P W 501 \backslash 16-F e b-2006 \backslash 0 \backslash \ \#$ b31 yp/6-31G(d) opt freq \\comment $\backslash 0,2 \backslash \mathrm{C}, 0.2317412779,-0.6454274385,-0.550899395 \backslash \mathrm{H}, 0.6627196918,-0.0585$ $847594,-1.3555811413 \backslash \mathrm{H}, 0.9123083101,-1.0753934279,0.1768311216 \backslash \mathrm{C},-1.1577631265,-0.841$ $568992,-0.4547222619 \backslash \mathrm{C},-2.0559798205,-0.2782144998,-1.4097435922 \backslash \mathrm{H},-1.6475608673,0.31$ $35288091,-2.2254162387 \backslash \mathrm{C},-1.7281592903,-1.6122988353,0.6021670535 \backslash \mathrm{H},-1.0659134263,-2$. $0528549812,1.3435580409 \backslash \mathrm{C},-3.0979778413,-1.8025734479,0.6916618956 \backslash \mathrm{H},-3.5075239177,-$ $2.3941710224,1.5065436121 \backslash \mathrm{C},-3.9584307316,-1.2374756078,-0.2610616619 \backslash \mathrm{H},-5.031500675$ 7,-1.3893653492,-0.1870271577\C,-3.424 1973797,-0.4751042738,-1.30996744\Н,-4.087042203 $1,-0.0357561738,-2.0511528352 \backslash \backslash$ Version $=I A 64 L-G 03 R e v D .01 \backslash$ State $=2-A \backslash H F=-270.9151434 \backslash \mathrm{S}$ $2=0.783684 \backslash \mathrm{S} 2-1=0 . \backslash \mathrm{S} 2 \mathrm{~A}=0.750769 \backslash \mathrm{RMSD}=5.422 \mathrm{e}-09 \backslash \mathrm{RMSF}=2.793 \mathrm{e}-05 \backslash \mathrm{Thermal}=0 . \backslash \mathrm{Dipole}=$ $-0.0518841,-0.0076017,0.0033884 \backslash \mathrm{PG}=\mathrm{C} 01[\mathrm{X}(\mathrm{C} 7 \mathrm{H} 7)] \backslash \backslash @$ 